\title{
UCRL-JRNL-221053
}

LAWRENCE LIVERMORE N A TIO NAL LABORATORY
Progress towards high performance plasmas in the National Spherical Torus Experiment (NSTX)

S. M. Kaye, et al.

May 3, 2006

Nuclear Fusion 
This document was prepared as an account of work sponsored by an agency of the United States Government. Neither the United States Government nor the University of California nor any of their employees, makes any warranty, express or implied, or assumes any legal liability or responsibility for the accuracy, completeness, or usefulness of any information, apparatus, product, or process disclosed, or represents that its use would not infringe privately owned rights. Reference herein to any specific commercial product, process, or service by trade name, trademark, manufacturer, or otherwise, does not necessarily constitute or imply its endorsement, recommendation, or favoring by the United States Government or the University of California. The views and opinions of authors expressed herein do not necessarily state or reflect those of the United States Government or the University of California, and shall not be used for advertising or product endorsement purposes. 


\section{Progress towards high performance plasmas in the National Spherical Torus Experiment (NSTX)}

S.M. Kaye ${ }^{1}$, M.G. Bell ${ }^{1}$, R.E. Bell ${ }^{1}$, S. Bernabei ${ }^{1}$, J. Bialek ${ }^{2}$, T. Biewer ${ }^{1}$, W. Blanchard ${ }^{1}$, J. Boedo ${ }^{3}$, C. Bush ${ }^{4}$, M.D. Carter ${ }^{4}$, W. Choe ${ }^{5}$, N. Crocker ${ }^{6}$, D.S. Darrow ${ }^{1}$, W. Davis ${ }^{1}$, L. Delgado-Aparicio ${ }^{7}$, S. Diem ${ }^{1}$, J. Ferron ${ }^{8}$, A. Field ${ }^{9}$, J. Foley ${ }^{1}$, E.D. Fredrickson ${ }^{1}$, D.A. Gates ${ }^{1}$, T. Gibney ${ }^{1}$, R. Harvey ${ }^{10}$, R.E. Hatcher ${ }^{1}$, W. Heidbrink ${ }^{11}$, K. Hill ${ }^{1}$, J.C. Hosea ${ }^{1}$, T.R. Jarboe ${ }^{12}$, D.W. Johnson ${ }^{1}$, R. Kaita ${ }^{1}$, C. Kessel ${ }^{1}$, S. Kubota ${ }^{6}$, H.W. Kugel ${ }^{1}$, J. Lawson ${ }^{1}$, B.P. LeBlanc ${ }^{1}$, K.C. Lee $^{13}$, F. Levinton ${ }^{14}$, R. Maingi ${ }^{4}$, J. Manickam ${ }^{1}$, R. Maqueda ${ }^{14}$, R. Marsala ${ }^{1}$, D. Mastrovito ${ }^{1}$, T.K. Mau ${ }^{3}$, S.S. Medley ${ }^{1}$, J. Menard ${ }^{1}$, H. Meyer ${ }^{9}$, D.R. Mikkelsen ${ }^{1}$, D. Mueller ${ }^{1}$, T. Munsat ${ }^{15}$, B.A. Nelson ${ }^{12}$, C. Neumeyer ${ }^{1}$, N. Nishino ${ }^{16}$, M. Ono ${ }^{1}$, H. Park ${ }^{1}$, W. Park ${ }^{1}$, S. Paul ${ }^{1}$, T. Peebles 6 , M. Peng ${ }^{4}$, C. Phillips ${ }^{1}$, A. Pigarov ${ }^{3}$, R. Pinsker ${ }^{8}$, A. Ram $^{17}$, S. Ramakrishnan ${ }^{1}$, R. Raman $^{12}$, D. Rasmussen ${ }^{4}$, M. Redi $^{1}$, M. Rensink ${ }^{18}$, G. Rewoldt ${ }^{1}$, J. Robinson ${ }^{1}$, P. Roney ${ }^{1}$, A.L. Roquemore ${ }^{1}$, E. Ruskov ${ }^{11}$, P. Ryan ${ }^{4}$, S.A. Sabbagh ${ }^{2}$, H. Schneider ${ }^{1}$, C.H. Skinner ${ }^{1}$, D.R. Smith ${ }^{1}$, A. Sontag ${ }^{2}$, V. Soukhanovskii ${ }^{18}$, T. Stevenson ${ }^{1}$, D. Stotler ${ }^{1}$, B. Stratton ${ }^{1}$, D. Stutman ${ }^{7}$, D. Swain ${ }^{4}$, E. Synakowski ${ }^{1}$, Y. Takase ${ }^{19}$, G. Taylor ${ }^{1}$, K. Tritz ${ }^{7}$, A. von Halle ${ }^{1}$, M. Wade ${ }^{4}$, R. White ${ }^{1}$, J. Wilgen ${ }^{4}$, M. Williams ${ }^{1}$, J.R. Wilson ${ }^{1}$, W. Zhu ${ }^{2}$, S.J. Zweben ${ }^{1}$, R. Akers ${ }^{9}$, P. Beiersdorfer ${ }^{18}$, R. Betti ${ }^{20}$, T. Bigelow ${ }^{4}$, M. Bitter ${ }^{1}$, P. Bonoli ${ }^{17}$, C. Bourdelle ${ }^{21}$, C.S. Chang ${ }^{22}$, J. Chrzanowski ${ }^{1}$, C. Domier ${ }^{13}$, L. Dudek ${ }^{1}$, P.C. Efthimion ${ }^{1}$, M. Finkenthal ${ }^{7}$, E. Fredd ${ }^{1}$, G.Y. Fu ${ }^{1}$, A. Glasser ${ }^{23}$, R.J. Goldston ${ }^{1}$, N.L. Greenough ${ }^{1}$, L.R. Grisham ${ }^{1}$, N. Gorelenkov ${ }^{1}$, L. Guazzotto ${ }^{20}$, R.J. Hawryluk ${ }^{1}$, J. Hogan ${ }^{4}$, W. Houlberg ${ }^{4}$, D. Humphreys ${ }^{8}$, F. Jaeger ${ }^{4}$, M. Kalish ${ }^{1}$, S. Krasheninnikov ${ }^{3}$, L.L. Lao ${ }^{8}$, J. Lawrence ${ }^{24}$, J. Leuer ${ }^{8}$, D. Liu ${ }^{11}$, N.C. Luhmann ${ }^{13}$, E. Mazzucato ${ }^{1}$, G. Oliaro ${ }^{1}$, D. Pacella ${ }^{25}$, R. Parsells ${ }^{1}$, M. Schaffer ${ }^{8}$, I. Semenov ${ }^{26}$, K.C. Shaing ${ }^{27}$, M.A. Shapiro ${ }^{17}$, K. Shinohara ${ }^{28}$, P. Sichta ${ }^{1}$, X. Tang ${ }^{23}$, R. Vero ${ }^{7}$, D. Walker ${ }^{8}$ and W. Wampler ${ }^{29}$

\footnotetext{
${ }^{1}$ Princeton Plasma Physics Laboratory, Princeton University, Princeton, NJ 08543, USA

2 Department of Applied Physics, Columbia University, NYC, NY, USA

${ }^{3}$ University of California, San Diego, CA, USA

${ }^{4}$ Oak Ridge National Laboratory, Oak Ridge, TN, USA

${ }^{5}$ Korea Advanced Institute of Science and Technology, Taejon, Korea

${ }^{6}$ University of California, Los Angeles, CA, USA

${ }^{7}$ Johns Hopkins University, Baltimore, MD, USA

${ }^{8}$ General Atomics, San Diego, CA, USA

${ }^{9}$ Euratom-UKAEA Fusion Associates, Abingdon, Oxfordshire, UK

${ }^{10}$ Compx, Del Mar, CA, USA

${ }^{11}$ University of California, Irvine, CA, USA

${ }^{12}$ University of Washington, Seattle, WA, USA

${ }^{13}$ University of California, Davis, CA, USA

${ }^{14}$ Nova Photonics, Princeton, NJ, USA

${ }^{15}$ University of Colorado, Boulder, CO, USA

${ }^{16}$ Hiroshima University, Hiroshima, Japan

${ }^{17}$ Massachusetts Institute of Technology, Cambridge, MA, USA

${ }^{18}$ Lawrence Livermore National Laboratory, Livermore, CA, USA

${ }^{19}$ University of Tokyo, Tokyo, Japan

${ }^{20}$ University of Rochester, Rochester, NY, USA

${ }^{21}$ CEA, Cadarache, France

${ }^{22}$ New York University, NYC, NY, USA
} 


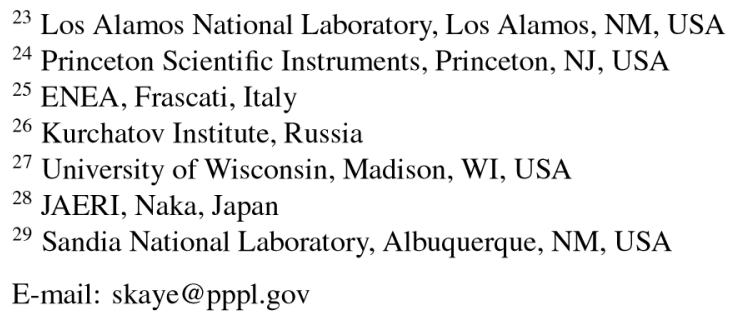

\begin{abstract}
The major objective of the National Spherical Torus Experiment (NSTX) is to understand basic toroidal confinement physics at low aspect ratio and high $\beta_{\mathrm{T}}$ in order to advance the spherical torus (ST) concept. In order to do this, NSTX utilizes up to $7.5 \mathrm{MW}$ of neutral beam injection, up to $6 \mathrm{MW}$ of high harmonic fast waves (HHFWs), and it operates with plasma currents up to $1.5 \mathrm{MA}$ and elongations of up to 2.6 at a toroidal field up to $0.45 \mathrm{~T}$. New facility, and diagnostic and modelling capabilities developed over the past two years have enabled the NSTX research team to make significant progress towards establishing this physics basis for future ST devices. Improvements in plasma control have led to more routine operation at high elongation and high $\beta_{\mathrm{T}}$ (up to $\sim 40 \%$ ) lasting for many energy confinement times. $\beta_{\mathrm{T}}$ can be limited by either internal or external modes. The installation of an active error field (EF) correction coil pair has expanded the operating regime at low density and has allowed for initial resonant EF amplification experiments. The determination of the confinement and transport properties of NSTX plasmas has benefitted greatly from the implementation of higher spatial resolution kinetic diagnostics. The parametric variation of confinement is similar to that at conventional aspect ratio but with values enhanced relative to those determined from conventional aspect ratio scalings and with a $B_{\mathrm{T}}$ dependence. The transport is highly dependent on details of both the flow and magnetic shear. Core turbulence was measured for the first time in an ST through correlation reflectometry. Non-inductive start-up has been explored using PF-only and transient co-axial helicity injection techniques, resulting in up to $140 \mathrm{kA}$ of toroidal current generated by the latter technique. Calculated bootstrap and beam-driven currents have sustained up to $60 \%$ of the flat-top plasma current in NBI discharges. Studies of HHFW absorption have indicated parametric decay of the wave and associated edge thermal ion heating. Energetic particle modes, most notably toroidal Alfvén eigenmodes and fishbone-like modes result in fast particle losses, and these instabilities may affect fast ion confinement on devices such as ITER. Finally, a variety of techniques has been developed for fuelling and power and particle control.
\end{abstract}

PACS numbers: 52.55.Fa, 52.55.Fi, 52.55.Dy, 52.55.Tn, 52.55.Wq

\section{Introduction}

The National Spherical Torus Experiment (NSTX) [1-3] is a low aspect ratio torus whose goals are to advance the understanding of toroidal confinement physics using the leverage of high $\beta_{\mathrm{T}}\left(=\langle p\rangle /\left(B_{\mathrm{T}}^{2} / 2 \mu_{0}\right)\right)$ and low aspect ratio. This understanding will help to develop the physics basis for advancing the spherical torus (ST) concept [4, 5]. In order to do this, an experimental database and a theoretical understanding must be developed in four areas: macroscopic plasma behaviour, transport, waves and energetic particles and plasma-boundary interfaces. NSTX goals in each area include:

(a) Macroscopic plasma behaviour: Attainment of wall stabilized plasmas with $\beta_{\mathrm{T}}$ up to $\sim 40 \%$ and normalized $\beta$, $\beta_{\mathrm{N}}$, up to 9 , compatible with non-inductive current start-up and sustainment. $\beta_{\mathrm{T}}$ is defined with respect to the vacuum toroidal magnetic field at the geometric axis;

(b) Transport: Routine access to long-pulse plasmas with confinement enhanced by at least $60 \%$ over L-mode values; (c) Waves and energetic particles: The ability to heat electrons selectively and drive current non-inductively using RF waves, and to achieve good confinement of energetic particles in the super-Alfvénic regime;

(d) Plasma-boundary interface: Density control and the ability to operate the compact ST configuration with acceptable heat flux to the plasma facing surfaces during steady-state operation and with transients.

NSTX is both addressing these issues individually and is integrating the conditions for each in order to develop steadystate, high performance plasmas. While the international ST programme is widely based and varied [6-8], NSTX is uniquely positioned to address the high performance issues described above. This paper presents the highlights of NSTX research over the last two years. The device capabilities and diagnostics are described in section 2 with emphasis on the new capabilities commissioned in 2003 to 2004 . Progress towards the main goals in each of the four physics areas mentioned above and towards developing integrated high-performance scenarios will be given in section 3 . A summary and discussion of future plans will be given in section 4. Particular emphasis 
will be placed on the new and unique results of the NSTX research programme.

\section{Device description}

NSTX operates at low aspect ratio with $R=0.85 \mathrm{~m}$ and $a=0.67 \mathrm{~m}(R / a \simeq 1.27)$. Over the past two years, NSTX has operated with plasma currents up to $1.4 \mathrm{MA}, B_{\mathrm{T}}$ up to $0.45 \mathrm{~T}$ and elongations up to 2.6 in both the lower single null (LSN) or double null diverted (DND) configurations. Close-fitting conducting plates provide passive stabilization of vertical motion and external MHD modes. Deuterium neutral beam power of just over $7 \mathrm{MW}$ at $100 \mathrm{keV}$ and high harmonic fast wave (HHFW) power of up to $6 \mathrm{MW}$ at $30 \mathrm{MHz}$ are sources of heating and non-inductive current drive. Plasma operation was primarily in deuterium, although helium was sometimes used during HHFW operation. The inner and outer halves of the vessel are isolated electrically for generating plasma current non-inductively through co-axial helicity injection (CHI).

Facility upgrades over the past two years included the installation and commissioning of a multi-barrel lithium pellet injector, a supersonic gas injector for localized and efficient fuelling, provision of a capacitor bank power supply for CHI experiments and modifications to the HHFW antenna feedthroughs for extending the performance reliability and power delivered to the plasma. A facility upgrade critical to the success of the majority of experiments run in the past year was reduced latency in the plasma control system from several milliseconds to under $1 \mathrm{~ms}$, improving the real-time plasma control and leading to more routine operation at high elongation [9]. Continued development of real-time EFIT (rtEFIT [10]) led to its successful use in experiments that required fine boundary control. An extra set of PF coils and power supplies was commissioned for use in non-solenoidal start-up experiments, and a pair of ex-vessel control coils for error field (EF) compensation, and ultimately for control of resistive wall modes (RWM), was installed and operated.

The past two years also saw improvements in the NSTX diagnostics critical to advancing our understanding of toroidal confinement physics. A new 51-channel charge exchange recombination spectroscopy (CHERS) diagnostic for measuring ion temperature, toroidal rotation velocity and carbon density profiles was installed. Additional new diagnostics included an edge rotation diagnostic (ERD) to measure simultaneously the poloidal and toroidal rotation, temperature and density of both $\mathrm{C}^{2+}$ and $\mathrm{He}^{+}$ions at the plasma edge, magnetic sensors in the divertor region to aid equilibrium reconstruction, in-vessel magnetic sensors mounted on the passive stabilizer plates and located approximately $5-10 \mathrm{~cm}$ outside the last closed flux surface for measuring the $n=$ 1-3 mode structures of external/RWM modes, and a high frequency Langmuir probe to measure fluctuations associated with RF heating. A scanning neutral particle analyser (NPA) was installed to measure the thermal and fast ion distribution functions and a microwave correlation reflectometer system was upgraded to measure long-wavelength turbulence in the plasma core. Edge fluctuations were measured with an upgraded reciprocating edge Langmuir probe [11], a new fast camera for gas puff imaging (GPI) measurements, a new divertor visible camera and edge channels in the FIR

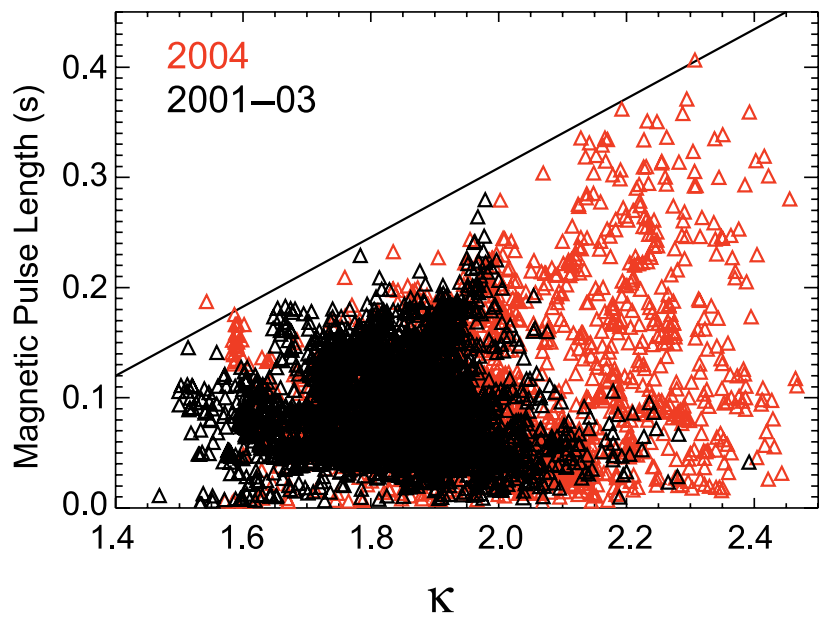

Figure 1. Magnetic pulse length versus elongation for the 2004 experimental campaign (red) and earlier years (black). The magnetic pulse length is defined as $\int_{I_{\mathrm{p}}>0.85 I_{\mathrm{p}, \max }}\left(I_{\mathrm{p}} \mathrm{d} t\right) / I_{\mathrm{TF}}$, where $I_{\mathrm{TF}}$ is the total toroidal field coil threading current.

interferometer system. Finally, an eight channel motional Stark effect (MSE) diagnostic was installed and has recently been used to determine the plasma current profile.

\section{Experimental results}

\subsection{Macroscopic plasma behaviour}

A recent technical improvement involved reducing the time response of the plasma control system from 4 to $0.75 \mathrm{~ms}$ [9]. This improved the feedback control for vertical stability, and it led to routine operation at higher elongation, $\kappa$, triangularity, $\delta$, and pulse lengths than those achieved in previous years. This is characterized by plotting the magnetic pulse length, $\tau_{\mathrm{p} \text {,mag }}$, as a function of $\kappa$ in figure 1. $\tau_{\mathrm{p} \text {,mag }}$ is defined as $\int_{I_{\mathrm{p}}>0.85 I_{\mathrm{p}, \max }}\left(I_{\mathrm{p}} \mathrm{d} t\right) / I_{\mathrm{TF}}$, where $I_{\mathrm{TF}}$ is the total toroidal field coil threading current. The weighting of $\tau_{\mathrm{p}, \operatorname{mag}}$ by the magnetic utilization factor, $I_{\mathrm{p}} / I_{\mathrm{TF}}$, normalizes out differences due to $q$, since $I_{\mathrm{p}} / I_{\mathrm{TF}} \sim 1 / q_{\text {cyl }}$ for fixed aspect ratio, $R / a$. The elongation was calculated by the magnetics-based equilibrium reconstruction code EFIT, adapted for use on NSTX [12,13]. The control system improvement permitted operation over an extended range of elongation; $\kappa$ values exceeding 2.6 at low plasma internal inductance, $l_{\mathrm{i}}(\sim 0.5)$, and $\delta$ up to 0.8 were obtained (not simultaneously). A $20 \%$ increase over the previous maximum elongation is seen.

The ability to access higher elongation more routinely benefits most operational scenarios in NSTX. In particular, the greater shaping allowed for higher plasma current at otherwise fixed conditions, leading to higher values of normalized current, $I_{\mathrm{p}} / a B_{\mathrm{T}}$, and hence higher values of $\beta_{\mathrm{T}}$. Here, the $B_{\mathrm{T}}$ used to define both $\beta_{\mathrm{T}}$ and $I_{\mathrm{p}} / a B_{\mathrm{T}}$ is the vacuum toroidal field at the geometric axis. Shown in figure 2 is a plot of peak $\beta_{\mathrm{T}}$ as determined from EFIT for a subset of discharges shown in figure 1 . The EFIT calculations were based on external magnetic measurements with the pressure profile shape constrained by the electron pressure as measured by the Thomson scattering diagnostic. The error bar is the 


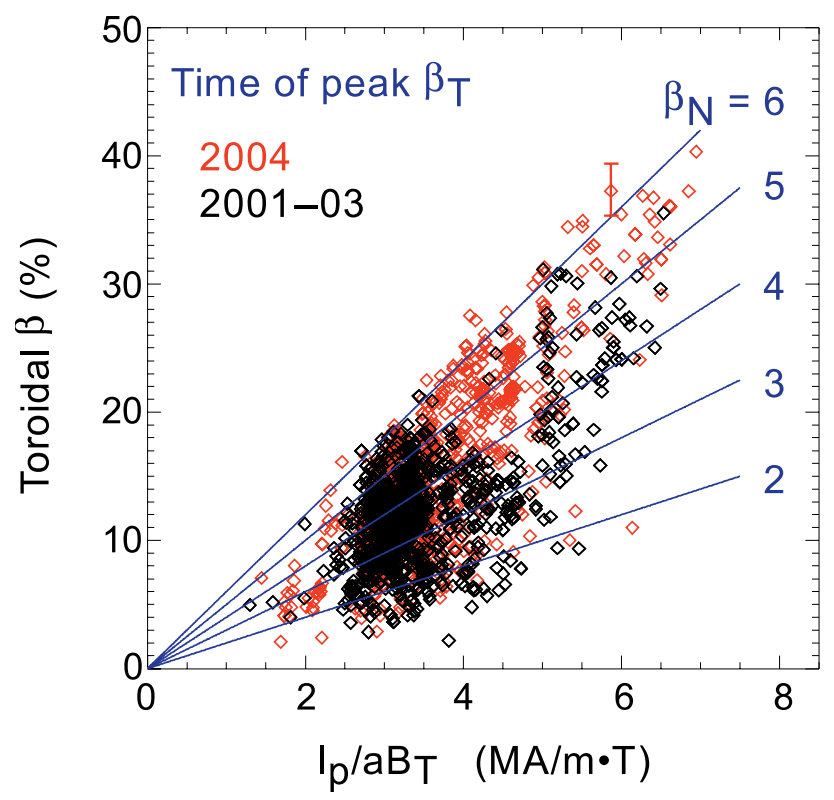

Figure 2. Toroidal $\beta$ versus $I_{\mathrm{p}} / a B_{\mathrm{T}}$ for 2004 experimental campaign (red) and earlier years (black). Here, $B_{\mathrm{T}}$ is the vacuum toroidal magnetic field at the geometric axis. The data are taken at the time of peak $\beta_{\mathrm{T}}$ in each discharge.

EFIT random uncertainty in the value of $\beta_{\mathrm{T}}$. Values of the normalized $\beta_{\mathrm{T}}, \beta_{\mathrm{N}}=\beta_{\mathrm{T}} /\left(I_{\mathrm{p}} / a B_{\mathrm{T}}\right)$, of up to $6.2 \% \mathrm{~m} \mathrm{~T} / \mathrm{MA}$ at the time of peak $\beta_{\mathrm{T}}$ were attained over the full range of $I_{\mathrm{p}} / a B_{\mathrm{T}}$. A maximum value of $\beta_{\mathrm{N}}=6.8 \% \mathrm{~m} \mathrm{~T} / \mathrm{MA}$ has been achieved at peak poloidal $\beta, \beta_{\mathrm{P}}=1.8$. The benefit of being able to achieve higher $\kappa$ and thus higher $I_{\mathrm{p}} / a B_{\mathrm{T}}$ is evidenced by significantly more high $\beta_{\mathrm{T}}(>30 \%$ ) shots during the 2004 experimental campaign than in previous years [14].

Not only was there more consistent attainment of high $\beta_{\mathrm{T}}$, but longer duration periods of high $\beta_{\mathrm{T}}$ were achieved as well. Figure 3 shows the time evolution of a $1 \mathrm{MA}$, high- $\beta_{\mathrm{T}}$ discharge. In this discharge, neutral beam injection starting early at $0.07 \mathrm{~s}$ and a short pause in the plasma current ramp-up at $0.09 \mathrm{~s}$ led to an early $\mathrm{L}-\mathrm{H}$ transition [15], as indicated by a sharp drop in the $\mathrm{D}_{\alpha}$ signal and the clear development of an edge transport barrier in the density profile. The TF was ramped down at $0.34 \mathrm{~s}$ after an edge localized mode (ELM)free period. During the ramp, the stored energy of the plasma plateaued at $250 \mathrm{~kJ}$ while $\beta_{\mathrm{T}}$ continued to increase. Also shown is the $\beta_{\mathrm{T}}$ evolution as computed by TRANSP, which is based on the measured kinetic profiles of the thermal ions and electrons and a Monte-Carlo calculation of the neutral beam ion component based on classical processes. The $\beta_{\mathrm{T}}$ values from the magnetic and kinetic calculations peak at $36 \%$ and $38 \%$, respectively. A significant result is that the EFIT value of $\beta_{\mathrm{T}}$ remained high ( $>30 \%$ ) for approximately $0.08 \mathrm{~s}$, which is about two energy confinement times for this discharge at this time. The earlier decrease in the TRANSP computed $\beta_{\mathrm{T}}$ is due to sparser time resolution of the kinetic profile measurements as compared to that of the magnetics. Also of significance is that while the central density continued to rise through the course of the discharge, the line-averaged density remained constant, as seen in the bottom panel of figure 3 , indicating control of the edge density due to the presence of ELMs.
This high- $\beta_{\mathrm{T}}$ discharge was limited by the growth of low- $n$ internal modes, as shown in figure 4 . The red traces in the figure show the evolution of the toroidal field, $\beta_{\mathrm{T}}$, and the magnetic fluctuation amplitude in the high- $\beta_{\mathrm{T}}$ discharge. The black traces are taken from a similar discharge, but one in which the toroidal field was held steady and, therefore, one with lower $\beta_{\mathrm{T}}(\simeq 20 \%)$. A long-duration $m / n=2 / 1$ mode in the mid-radius region existed in both discharges. A $1 / 1$ mode became unstable in the high- $\beta_{\mathrm{T}}$ discharge, as reflected by the increase in the amplitude of the magnetic fluctuations, at about $560 \mathrm{~ms}$. Rotation and diamagnetic effects were important in the high $-\beta_{\mathrm{T}}$ discharge in leading to a saturation rather than a complete reconnection of the $1 / 1$ mode, thus allowing $\beta_{\mathrm{T}}$ to increase to values over $35 \%$ [16]. The $1 / 1$ mode coupled and phase locked to the $2 / 1$ mode resulting in a reduction of the plasma rotation (figure 5 , top panel). The $\beta_{\mathrm{T}}$ started to decrease as the modes coupled, gradually at first, but then it collapsed as the mode frequency decreased through a critical value of $2 \mathrm{kHz}$ at $590 \mathrm{~ms}$. The plasma rotation remained high in the discharge with no TF ramp-down (figure 5, bottom panel).

RWMs were the $\beta_{\mathrm{T}}$-limiting mechanism in the low $q$ regime, where these modes were more prevalent [17]. The prevalence of these modes at low $q$ follows from the critical rotation frequency for mode growth, which was observed to scale as $1 / q^{2}$, with $q$ being the local value. The dynamics of a high- $\beta_{\mathrm{T}}$ discharge limited by a RWM are shown in figure 6 . The $\beta_{\mathrm{N}}$ of this discharge, shown in the top panel, exceeds a value of $5 \% \mathrm{~m} \mathrm{~T} / \mathrm{MA}$, which is about $10 \%$ greater than the no-wall limit computed by the DCON stability code [18], for many wall times $\left(\tau_{\text {wall }} \sim 5 \mathrm{~ms}\right)$. The in-vessel magnetic sensors show nearly simultaneous growth of $n=1-3$ modes, consistent with the DCON result indicating unstable $n=1-3$ RWM components. In this discharge, the mode was purely growing with no evidence of mode rotation down to $200 \mathrm{~Hz}$ and no higher frequency rotating tearing modes (bottom two panels).

In order to expand the NSTX operating space and allow for further increases in $\beta_{\mathrm{T}}$, it is essential to explore means by which performance-limiting MHD modes can be stabilized. This exploration was initiated using the first of three pairs of EF compensation/RWM control coils. The other two pairs will be commissioned for the next experimental campaign. The active control coil pair was used to eliminate low-density locked modes which could otherwise limit the potential for achieving high performance plasmas, as well as to understand the effect of the applied radial magnetic fields on modes at higher density and $\beta_{\mathrm{T}}$. In the appropriate polarity with $1 \mathrm{kA}-$ turn, corresponding to an applied $n=1 B_{\text {radial }} \sim 10 \mathrm{G}$ at the outer midplane, the threshold density for locked modes was reduced from 1.2 to $\sim 0.5 \times 10^{19} \mathrm{~m}^{-3}$. Experiments during the next campaign will focus on using the EF/RWM coil to suppress the low density locked modes and the RWM simultaneously [17].

Non-inductive operation will be essential for future STs because of space and neutron loading limitations. Several techniques of non-solenoidal plasma start-up are being explored on NSTX. In initial experiments in one technique, plasmas were pre-ionized using HHFW and ECH $(18 \mathrm{GHz})$ on the low field side of the vessel near the RF antenna. The currents in the outer poloidal field coils were adjusted to 

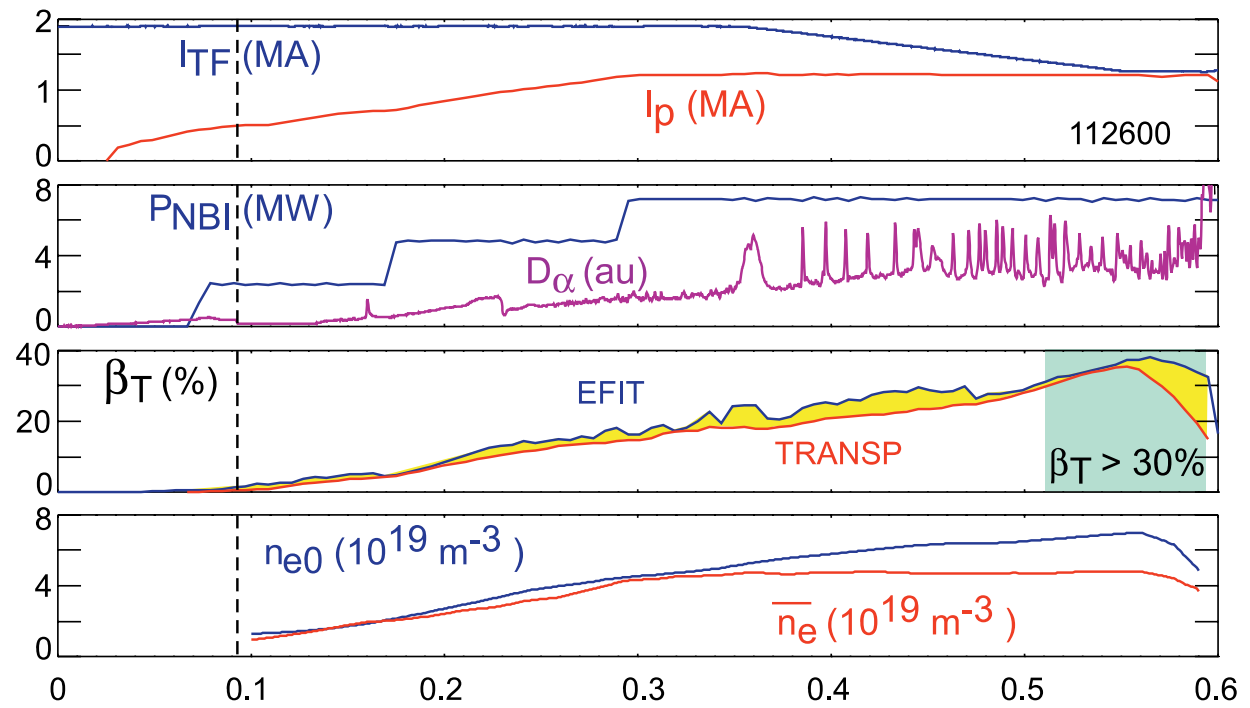

Time (s)

Figure 3. Temporal evolution of high- $\beta_{\mathrm{T}}$ discharge. $\kappa=2.4$ for this discharge. The time of the $\mathrm{L}-\mathrm{H}$ transition is denoted by the dashed vertical line.
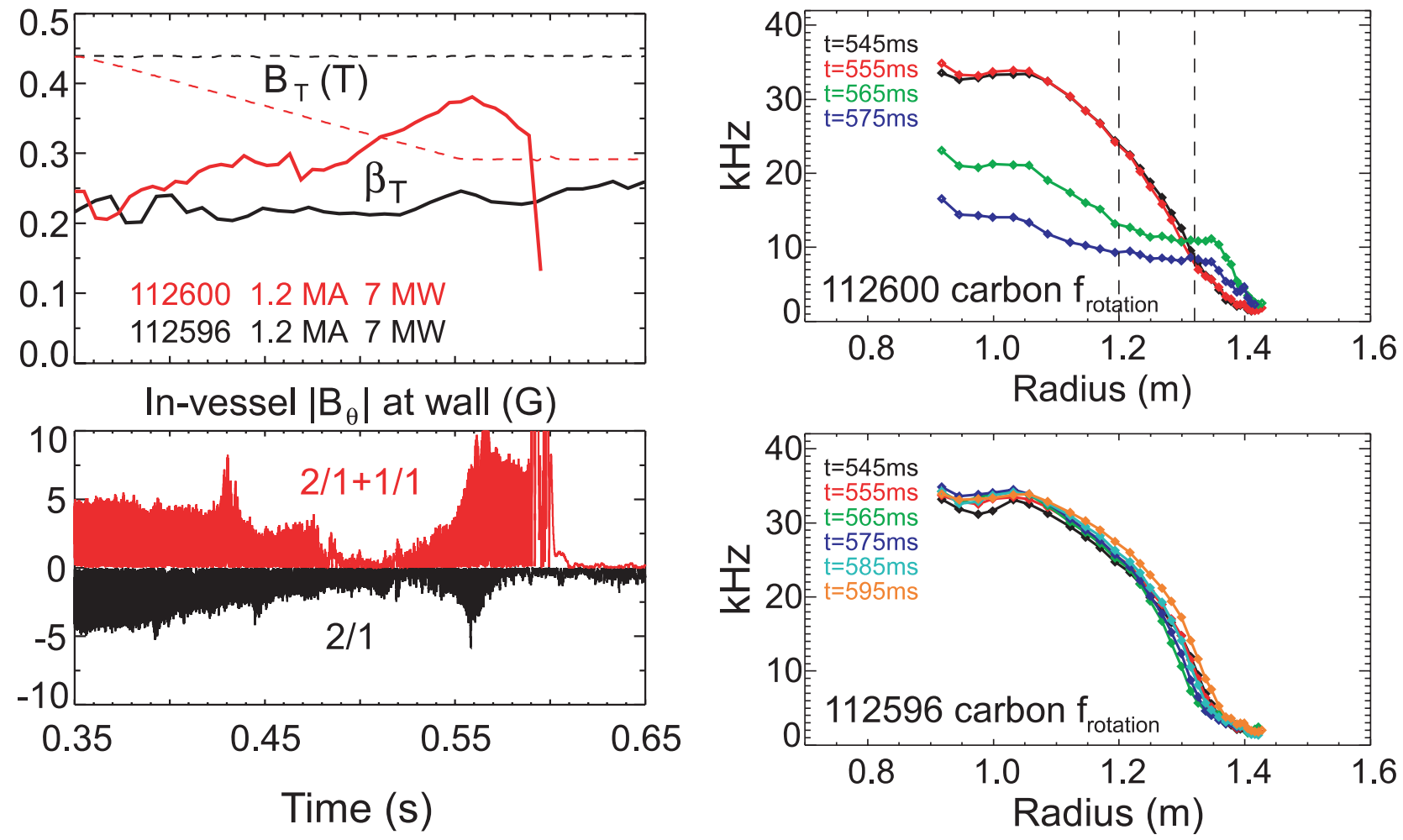

Figure 4. Comparison of $\beta_{\mathrm{T}}$ and mode evolution for two discharges, 112600 and 112596.

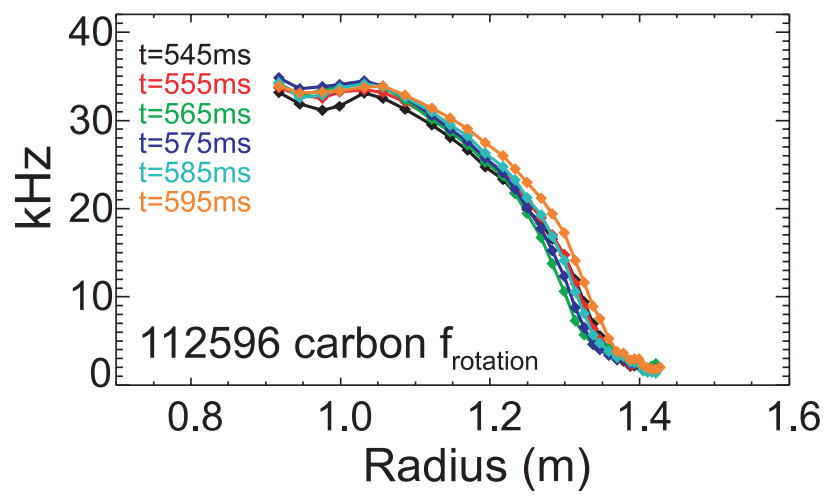

Figure 5. Evolution of carbon toroidal rotation profiles for the high- $\beta_{\mathrm{T}}$ discharges shown in figure 4.

establish a high quality field null $\left(B_{\phi} E_{\phi} / B_{\theta}>0.1 \mathrm{kV} \mathrm{m}^{-1}\right)$ over a region near the RF antenna, and then they were ramped to produce a toroidal loop voltage of 5-15 $\mathrm{V}$ near the antenna. Currents up to $20 \mathrm{kA}$ were produced, but the plasmas terminated on the centre stack. The goal for future work using this technique is to control the radial position of the nascent plasma to confine it to the region where the loop voltage is high, and thus achieve higher current.

Another technique that was tested is transient CHI [7], in which a pulse of voltage lasting for only a few milliseconds is applied between the inner and outer vessel segments, producing a plasma which is then propelled into the main chamber. A toroidal current is created in the presence of a toroidal field, and flux surfaces are believed to become closed due to reconnection processes associated with low- $n$ magnetic activity. The transient $\mathrm{CHI}$ technique has the benefit of reduced 
Progress towards high performance plasmas in NSTX

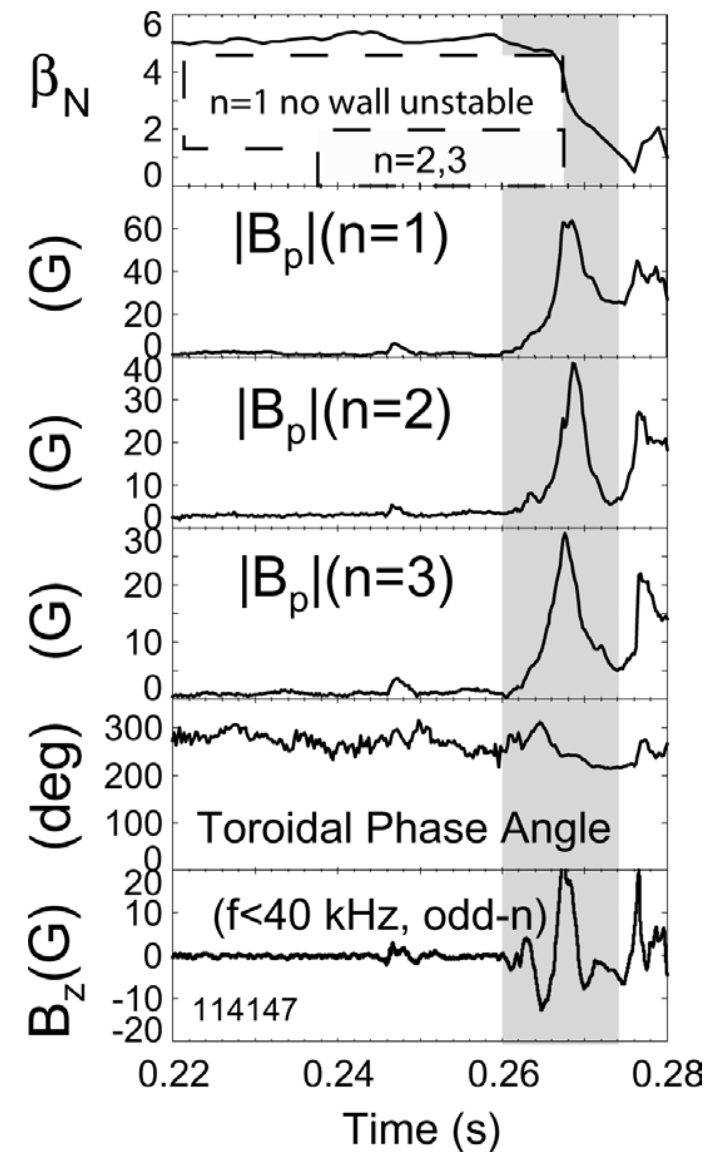

Figure 6. $\beta_{\mathrm{N}}=5.5$ plasma (top panel) limited by growth of a RWM. Shown are the $n=1-3$ components of the purely growing mode, and the mode phase and fluctuation amplitude of the Mirnov coil data (bottom two panels).

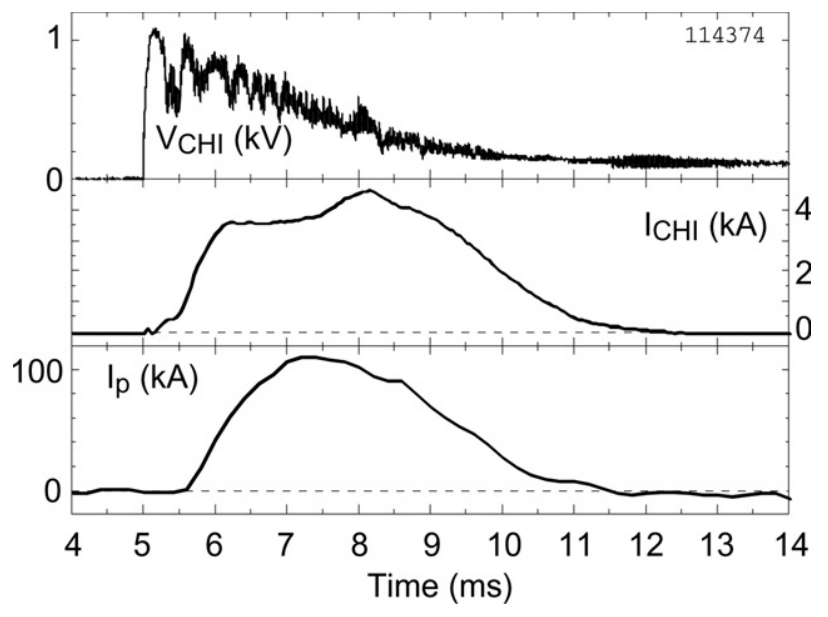

Figure 7. Early time evolution of a discharge whose toroidal current was generated by $\mathrm{CHI}$. We plot the $\mathrm{CHI}$ voltage and injector current, and the plasma current.

power to the walls, since the $\mathrm{CHI}$ is on for only a short time. An example of such a CHI discharge in NSTX is shown in figure 7. A voltage of $1 \mathrm{kV}$ was applied across the electrodes, generating an injector current of $4 \mathrm{kA}$ and a resulting plasma current of $100 \mathrm{kA}$ in this case, although the plasma current lasted only as long as the injector current. Plasma currents up to $140 \mathrm{kA}$ with amplification factors $\left(I_{\mathrm{p}} / I_{\mathrm{CHI}}\right)$ of up to 40 were achieved. This amplification factor is a factor of 2 greater than that obtained previously with longer duration $\mathrm{CHI}$ application. For the discharge shown in figure $7, T_{\mathrm{i}}$ and $T_{\mathrm{e}}$ of $20-25 \mathrm{eV}$ were measured. Flux closure is presently being assessed. Future experiments will focus on maintaining plasma current beyond the duration of the injector current in order to couple the plasma to other current drive sources, both inductive and non-inductive.

\subsection{Transport}

H-mode operation in NSTX resulted in the highest performance plasmas, with stored energies reaching close to $400 \mathrm{~kJ}$ in $1 \mathrm{MA}$ plasmas with $\sim 7 \mathrm{MW}$ of NB heating power. An experiment to study the $\mathrm{L}-\mathrm{H}$ threshold power was conducted as part of an NSTX/MAST identity experiment. The threshold power in NSTX was found to be low, $P_{\mathrm{NBI}} \simeq$ $350 \mathrm{~kW}$, in balanced DND plasmas at $0.5 \mathrm{MA}$ and $0.45 \mathrm{~T}$, with the threshold increasing to between 1 and $2 \mathrm{MW}$ in LSN plasmas (with the ion $\nabla B$ drift towards the X-point); these threshold powers are consistent with those on MAST for similar configurations and parameters [19]. Ohmic H-modes were often observed, most reproducibly at $B_{\mathrm{T}}>0.4 \mathrm{~T}$.

The confinement trends in NSTX were similar to those at conventional aspect ratio in some respects, but differed in others. Systematic scans of LSN H-mode plasmas at fixed power and $B_{\mathrm{T}}$ indicated a linear increase in both the global and thermal $\tau_{E}$, computed by EFIT and TRANSP, respectively, as a function of plasma current (0.6-1.2 MA). Figure 8 shows the results of this scan; the linear increase of total stored energy with plasma current is seen as is an increase in the electron stored energy, $W_{\mathrm{e}}$, as measured by the Thomson scattering diagnostic. The electron density was seen to vary by approximately $30 \%$ over the range of currents, but the central electron temperature remained constant. The 'ears' on the density profile reflect the buildup of carbon at the edge during the early and mid H-mode phases. A similar carbon density buildup at the edge, but not to the extent observed on NSTX, was seen at conventional aspect ratio in DIII-D VH-modes [20].

Results taken from these systematic scans, as well as from other discharges with similar operating parameters, indicate that at a fixed current of $0.8 \mathrm{MA}$, and $0.45 \mathrm{~T}$, the global and thermal $\tau_{E}$ were found to scale as $P^{-0.40}$ and $P^{-0.57}$, respectively, a slightly weaker degradation than at higher $R / a$. The current and power dependences for the thermal confinement time are shown in figure 9 . In contrast to the conventional aspect ratio, however, a $B_{\mathrm{T}}$ dependence was observed. This trend in the global and thermal confinement times is shown in figure 10. The left panel shows the global $\tau_{E}$ normalized to the $97 \mathrm{~L}$-mode scaling [21], and the right panel shows the thermal $\tau_{E}$ normalized to the H-mode ITERH$98 \mathrm{P}(\mathrm{y}, 2)$ thermal $\tau_{E}$ scaling [22]. The figures show that the global $\tau_{E}$ values are enhanced over the L-mode value, with enhancement factors of close to 2.8 at the highest $B_{\mathrm{T}}$ for both L- and H-mode plasmas. Although the confinement enhancements for L- and H-mode discharges are comparable, the L-mode discharges were much more transient than were the H-modes. 

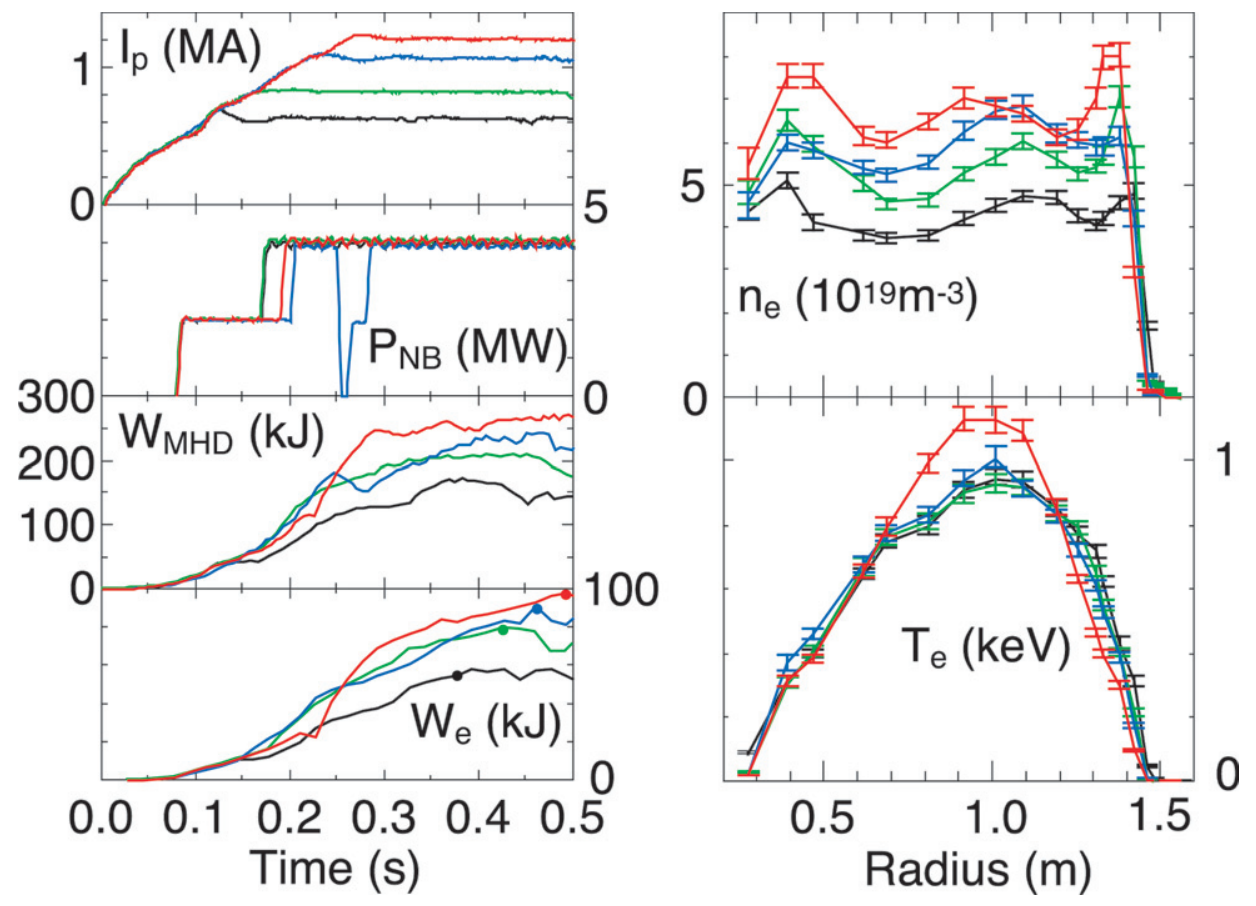

Figure 8. Plasma and electron stored energy evolutions for discharges from a systematic current scaling experiment at fixed neutral beam power. Also shown are the electron temperature and density profiles for these discharges at the time of peak electron stored energy.
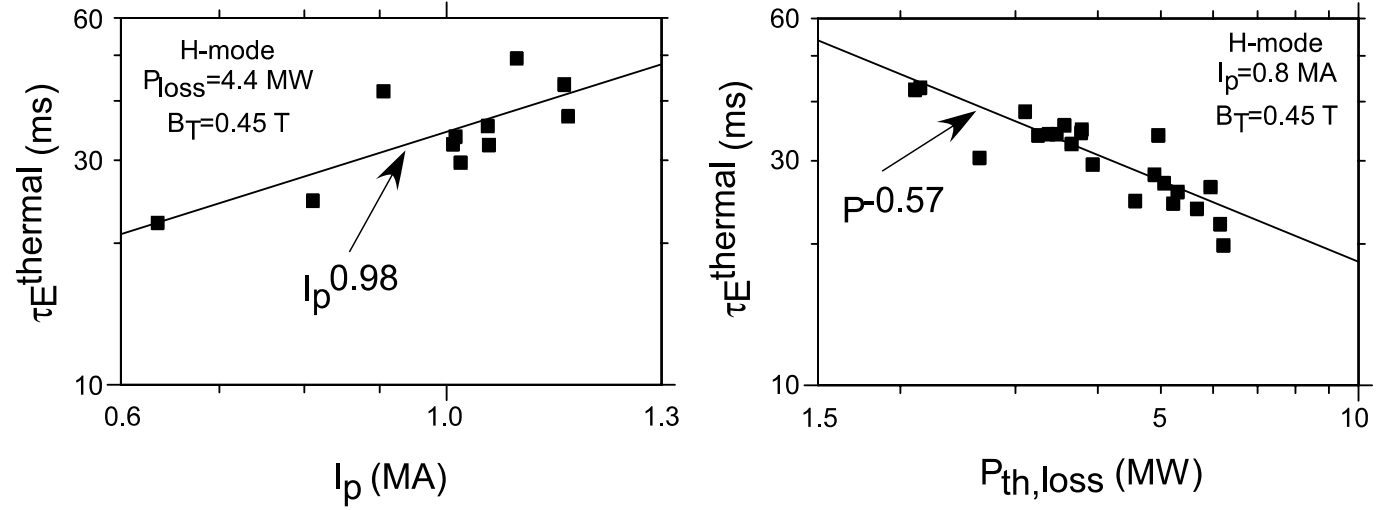

Figure 9. Dependence of thermal energy confinement time on $I_{\mathrm{p}}$ at fixed $B_{\mathrm{T}}$ and input power (left panel) and on heating power at fixed $I_{\mathrm{p}}$ and $B_{\mathrm{T}}$ (right panel). $P_{\mathrm{th}, \text { loss }}$ is the total input heating power minus $\mathrm{d} W / \mathrm{d} t$ and fast ion losses through charge-exchange, bad orbits and shine-through.
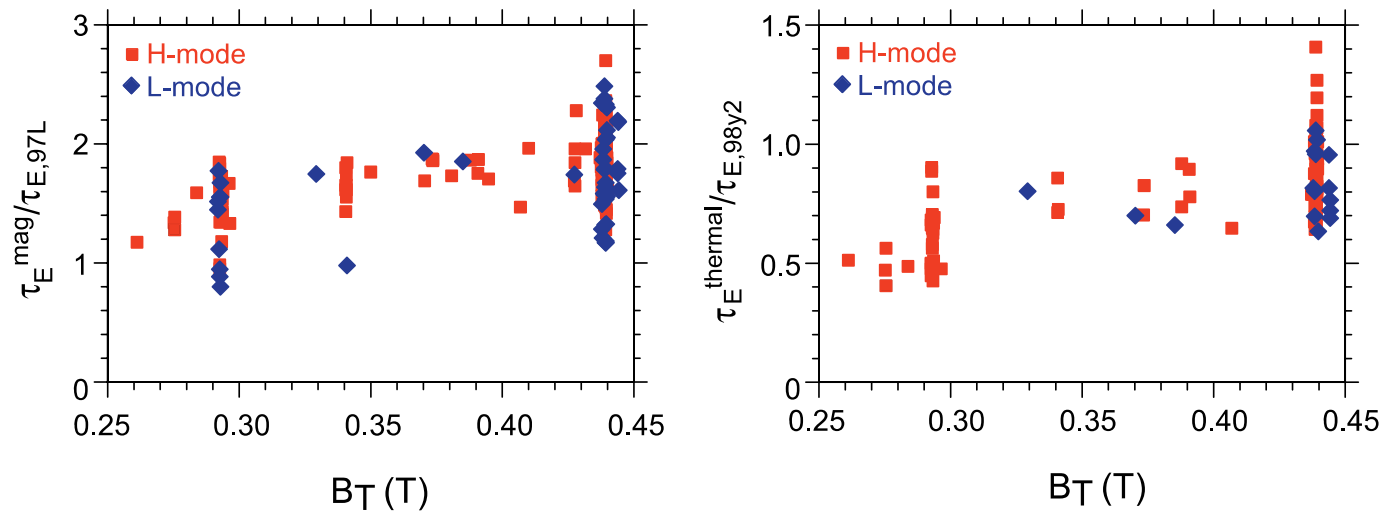

Figure 10. Global energy confinement time normalized to the $97 \mathrm{~L}$-mode scaling value (left panel) and thermal confinement time normalized to the $98 \mathrm{P}(\mathrm{y}, 2)$ scaling (right panel) plotted as a function of toroidal field. 
Progress towards high performance plasmas in NSTX

The thermal confinement enhancement factors are more modest, reaching 1.4 at the highest $B_{\mathrm{T}}$ for $\mathrm{H}$-mode plasmas, but with lower values at lower fields. Calculation uncertainties in the thermal $\tau_{E}$ are approximately $25 \%$. The thermal $\tau_{E}$ values ranged from a factor of 0.36 to 1.13 of the global $\tau_{E}$, with a mean value of 0.66 . The quality of the kinetic data at the lowest $B_{\mathrm{T}}$ precluded calculating the thermal $\tau_{E}$ at these fields with confidence. A reduction in confinement enhancement at the lowest $B_{\mathrm{T}}(<0.3 \mathrm{~T})$ is seen for both the global and thermal values. It is also seen that there is much scatter in the confinement enhancements at all fields. This is partly due to the fact that both ELMing and ELM-free discharges are shown in this comparison, with ELMing discharges having energy confinement time enhancement factors approximately 10-20\% below those of the ELM-free discharges. The dependence on $B_{\mathrm{T}}$ is also manifest statistically as a degradation of both $B_{\mathrm{T}} \tau_{E}$ and $B_{\mathrm{T}} \tau_{E \text {,th }}$ with $\beta_{\mathrm{T}}$.

Insight into the possible $B_{\mathrm{T}}$ dependence and processes causing transport can be gained by examining preliminary turbulence measurements using fixed-frequency $(30,42$ and $49 \mathrm{GHz}$ ) quadrature and swept-frequency $(26-40 \mathrm{GHz})$ homodyne correlation reflectometry systems. For the first time in NSTX, and in an ST, quantitative long-wavelength $\left(k_{\theta} \rho_{\mathrm{i}}<1\right)$ turbulence measurements have been made in the core $(r / a=0.2-0.7)$ of beam-heated L-mode plasma discharges. Correlation reflectometry data indicate radial correlation lengths $\left(L_{\mathrm{c}}\right)$ ranging from 2 to $25 \mathrm{~cm}$ with significantly smaller values observed in the outer plasma $(r / a \sim 0.65)$. Long wavelength electrostatic modes are expected to be stable or suppressed by $E \times B$ flow shear in the core. The longer correlation lengths measured at $r / a \sim 0.3-0.5$ may, therefore, be indicative of the existence of turbulence contributions from shorter scale fluctuations or, possibly, from the effect of beam-driven magnetic instabilities.

The correlation lengths measured in the outer plasma at $r / a=0.7$ (where ITG turbulence can exist) are illustrated in the top panel of figure 11 as a function of local $|B|$ during a fixed edge $q$ scan. As can be seen, correlation lengths are observed to increase with decreasing field, reaching values of approximately $8 \mathrm{~cm}$ at the lowest field. The bottom panel of figure 11 demonstrates that the correlation lengths scale with $\rho_{\mathrm{s}}\left(\rho_{\mathrm{s}} \propto\left(T_{\mathrm{e}} m_{\mathrm{i}}\right)^{1 / 2} / B_{\mathrm{T} 0}\right)$, with $L_{\mathrm{c}} / \rho_{\mathrm{s}}$ being $\sim 8$ over this range of fields. This is consistent with previous turbulence data from DIII-D L-mode plasmas as well as with associated nonlinear gyrokinetic code calculations based on ITG turbulence [23]. Quadrature reflectometry measurements taken for a similar set of discharges show a reduction in the measured reflectometer phase fluctuation level (for frequencies $>10 \mathrm{kHz}$ and ignoring large coherent modes) as the magnetic field is increased. Quantitative estimates of the density fluctuation levels using the measured phase depend on the details of the specific model employed. However, for these measurements all models would indicate a significant reduction in density fluctuation levels at higher magnetic fields. These general observations are consistent with poorer confinement at lower $B_{\mathrm{T}}$. In the context of core turbulence and transport, the data suggest that NSTX plasmas may represent a transition away from a long-wavelength, electrostatic turbulence dominated core to one where magnetic fluctuations or possibly shorter wavelength electrostatic turbulence play significant roles.
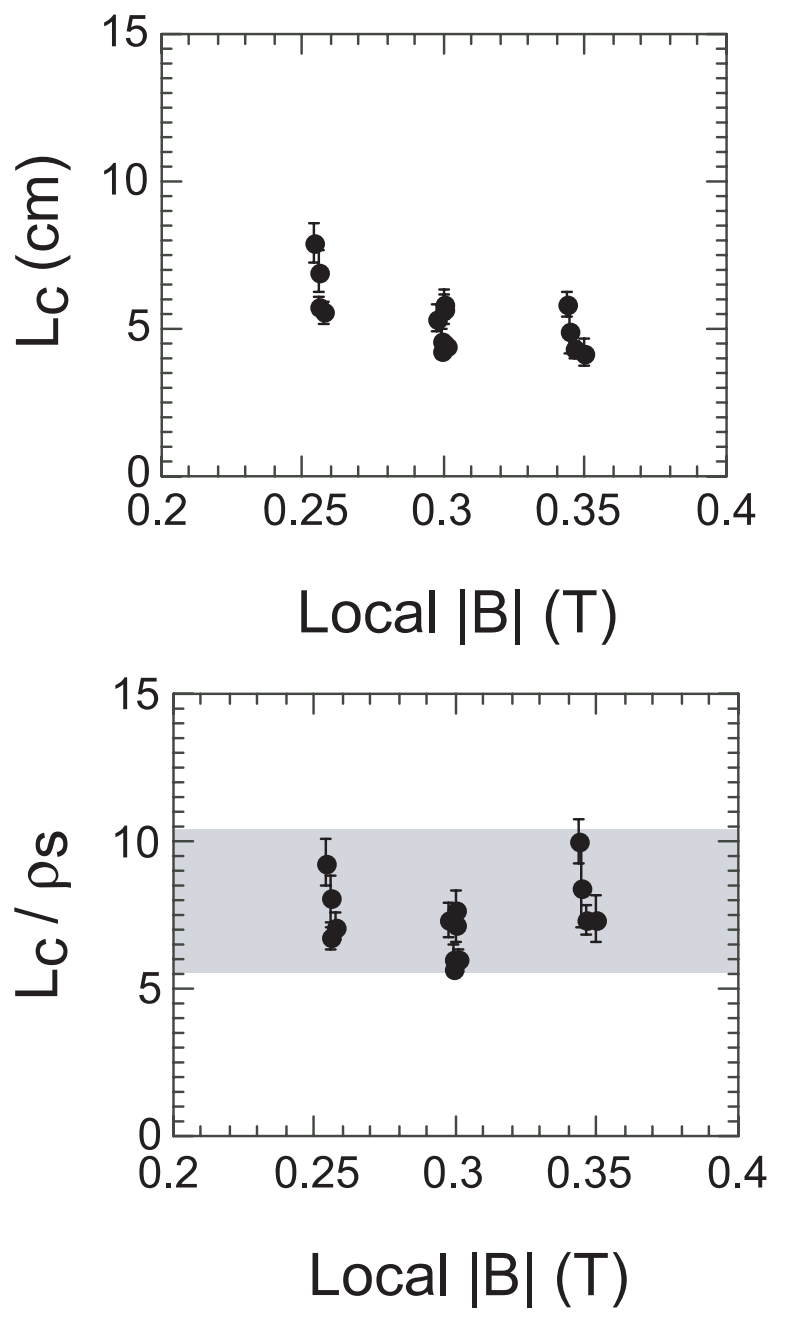

Figure 11. Reflectometer fluctuation radial correlation length as a function of local magnetic field (top) at $r / a=0.7$ for a toroidal field scan at fixed- $q$. Radial correlation length normalized to the ion characteristic gyroradius, $\rho_{\mathrm{s}}$ (bottom). The local $|B|$ is the local total magnetic field.

The determination of the transport properties of NSTX plasmas by TRANSP has benefitted greatly by the increased number of spatial points of the CHERS diagnostic. The calculations indicate, as before [24], that the electron channel dominates the transport loss in most $\mathrm{H}$-modes $\left(\chi_{\mathrm{e}}=10\right.$ $\left.20 \mathrm{~m}^{2} \mathrm{~s}^{-1}\right)$, resulting in $T_{\mathrm{e}}(0)$ values $\leqslant 2 \mathrm{keV}$, despite neutral beam heating powers of up to $7 \mathrm{MW}$. The ion thermal diffusivity is near or above the NCLASS [25] neoclassical value in many cases $\left(\chi_{\mathrm{i}}=1-5 \mathrm{~m}^{2} \mathrm{~s}^{-1}\right)$. NCLASS neoclassical values do not take into account enhancements to the neoclassical diffusivity by up to a factor of 2 due to finite orbit effects [26]. In the L-mode, $\chi_{\mathrm{i}} \approx \chi_{\mathrm{e}}\left(1-10 \mathrm{~m}^{2} \mathrm{~s}^{-1}\right)$ for line-averaged densities $\leqslant 4 \times 10^{19} \mathrm{~m}^{-3}$, but $\chi_{\mathrm{e}}>\chi_{\mathrm{i}}$ for higher densities.

The local transport properties of NSTX plasmas appear to be sensitive to variations in magnetic shear, as is shown by comparing two discharges with different $q$-profiles [27]. The $q$-profiles of these discharges were varied by changing the current ramp rate and the NBI timing in low density $\left(n_{\mathrm{e} 0} \sim 2 \times 10^{19} \mathrm{~m}^{-3}\right)$ L-mode discharges. In a discharge with a fast $I_{\mathrm{p}}$ ramp and early NBI, the $T_{\mathrm{i}}$ and $T_{\mathrm{e}}$ exhibited much 

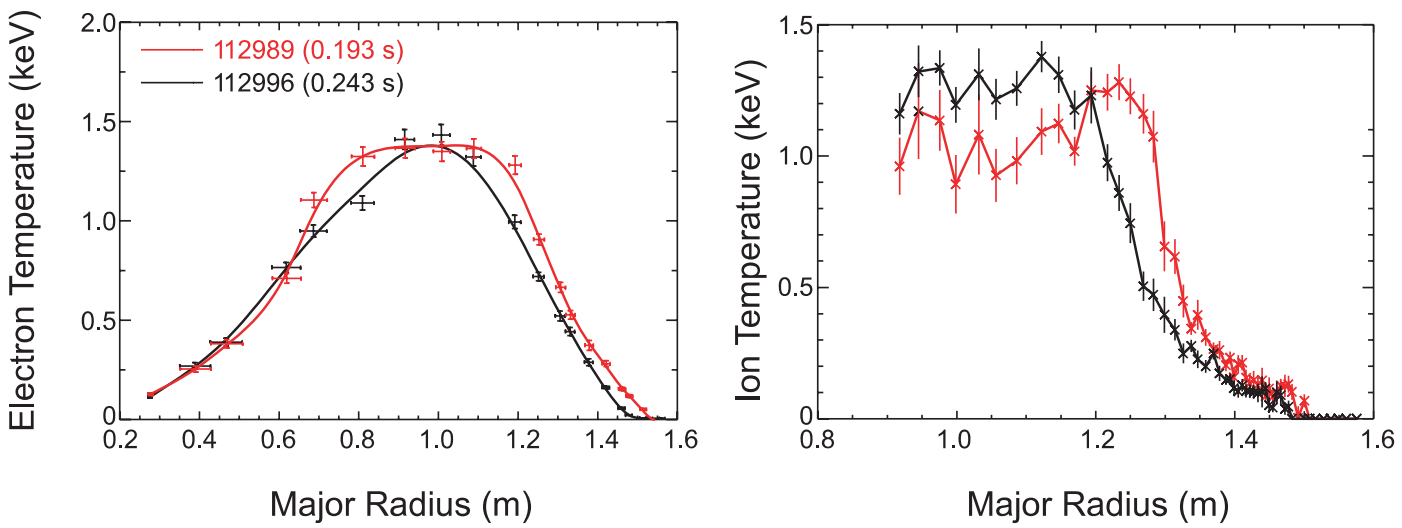

Figure 12. Electron and ion temperature profiles for discharges 112989 (fast $I_{\mathrm{p}}$ ramp and early NBI) and 112996 (slow $I_{\mathrm{p}}$ ramp and later $\mathrm{NBI}$ ) at times of comparable density and rotation.

stronger gradients near $r / a=0.5$ than in a discharge with a slower $I_{\mathrm{p}}$ ramp and later NBI, signifying the development of an internal transport barrier. The electron and ion temperature profiles from these discharges at times of comparable density and rotation velocity profiles are shown in figure 12, and the sharper temperature profile gradients can be seen in discharge 112989, the one with the faster $I_{\mathrm{p}}$ ramp and earlier neutral beam injection. The $q$-profiles for these two discharges, as determined in TRANSP assuming Sauter neoclassical resistivity [28], is shown in the top panel of figure 13. The modelling for the slow ramp/late NBI discharge (112996) shows a monotonic $q$-profile, while that for the fast ramp/early NBI discharge (112989) exhibits a magnetic shear reversal from $r / a=0.2$ to 0.5 . The electron transport barrier generally falls in the region of maximum negative magnetic shear, while the ion transport barrier coincides with the location of $q_{\min }$. MSE measurements of the current profile to confirm the reversed shear were not available for these discharges. It is noted, however, that off-axis $1 / 1$ modes were observed in 112989, and similar modes were observed in discharges in which equilibrium reconstructions that used MSE measurement as a constraint did indicate reversed shear. The implications of the possible reversed shear are seen in the bottom panels of figure 13, which show a reduction by a factor of 3-7 in the thermal diffusivities of both the electrons and ions in the region of reversed and low shear, respectively. $\chi_{\mathrm{i}}=\chi_{\mathrm{e}}$ outside this region. Because of uncertainties in $T_{\mathrm{e}}, T_{\mathrm{i}}$ and their gradients, the thermal diffusivities are highly uncertain in the shaded region, $r / a<0.2$ and $>0.7$.

Reflectometer measurements indicated both longer turbulence correlation lengths and higher estimated density fluctuation levels in the discharge with monotonic shear as compared to the one with possible reversed shear. GS2 $[29,30]$ gyrokinetic calculations indicate linear growth rates for micro-instabilities in the $k_{\theta} \rho_{\mathrm{s}}=0.1-10$ range (ITG/TEM, microtearing) near $r / a=0.45$, which are significantly higher in the monotonic than in the reversed shear case. In addition, high $k_{\theta} \rho_{\mathrm{s}}$ modes (ETGs) are suppressed in the region of reversed or low shear, supporting the general conclusion of the importance of electron physics in determining the overall confinement in NSTX [27]. Non-linear gyrokinetic calculations are underway to confirm these results and to study the stabilizing effect of sheared rotation.

\subsection{Waves and energetic particles}

The $30 \mathrm{MHz}$ (ninth harmonic of the deuterium gyrofrequency on-axis) system provides the potential for heating electrons selectively to reduce Ohmic flux consumption and for providing non-inductive current drive directly. The twelvestrap HHFW antenna has the capability to launch waves over a range of toroidal wavenumbers $\left(k_{\mathrm{T}}=3-14 \mathrm{~m}^{-1}\right)$ and directions. While significant electron heating has been observed in low density deuterium and helium plasmas, the actual power absorption of the electrons was found to depend sensitively on the spectrum of launched waves. Both the absorption and incremental confinement times in these HHFWonly plasmas were determined from the temporal response of the total stored energy to these modulations. The fractional electron power absorption determined from the responses is $80 \%$ at $k_{\mathrm{T}}=14 \mathrm{~m}^{-1}, 75 \%, 40 \%$ at $7 \mathrm{~m}^{-1},-7 \mathrm{~m}^{-1}$, respectively (counter, co-current injection) and $10 \%$ at $3 \mathrm{~m}^{-1}$, with the remainder of the power unaccounted for. Electron heating profiles are consistent with model calculations that predict broader heating profiles for higher $k_{\mathrm{T}}$, but the increment in electron stored energy is less than what would be expected for pure electron heating.

Heating of the edge thermal ions during HHFW was measured by the ERD [31], and this heating is being considered as a possible explanation for the apparent deficit in electron heating. This edge measurement indicates that the edge ions could be described as a two-temperature component plasma, with a significant hot component whose temperatures scaled with the HHFW power, and which could reach $0.6 \mathrm{keV}$, as shown in figure 14. The edge ion heating was associated with parametric decay of the launched HHFW wave as measured by a Langmuir probe in the plasma scrape-offlayer. A frequency spectrum of the probe signal is shown in figure 15; the fundamental wave at $30 \mathrm{MHz}$ is seen along with sidebands separated by $f_{\mathrm{c}, \mathrm{D}}$, indicative of a decay into an ion Bernstein wave (IBW). More IBW sidebands are observed with increasing $P_{\mathrm{HHFW}}$. It is also noted that a significant amount of HHFW power could be absorbed by fast ions in HHFW + NBI experiments.

The reduced HHFW power absorption limited the current driven by the HHFW, especially at $k_{\mathrm{T}}=3 \mathrm{~m}^{-1}$ where the current drive is predicted by theory to be maximal. Because of the importance of non-inductive current drive in 

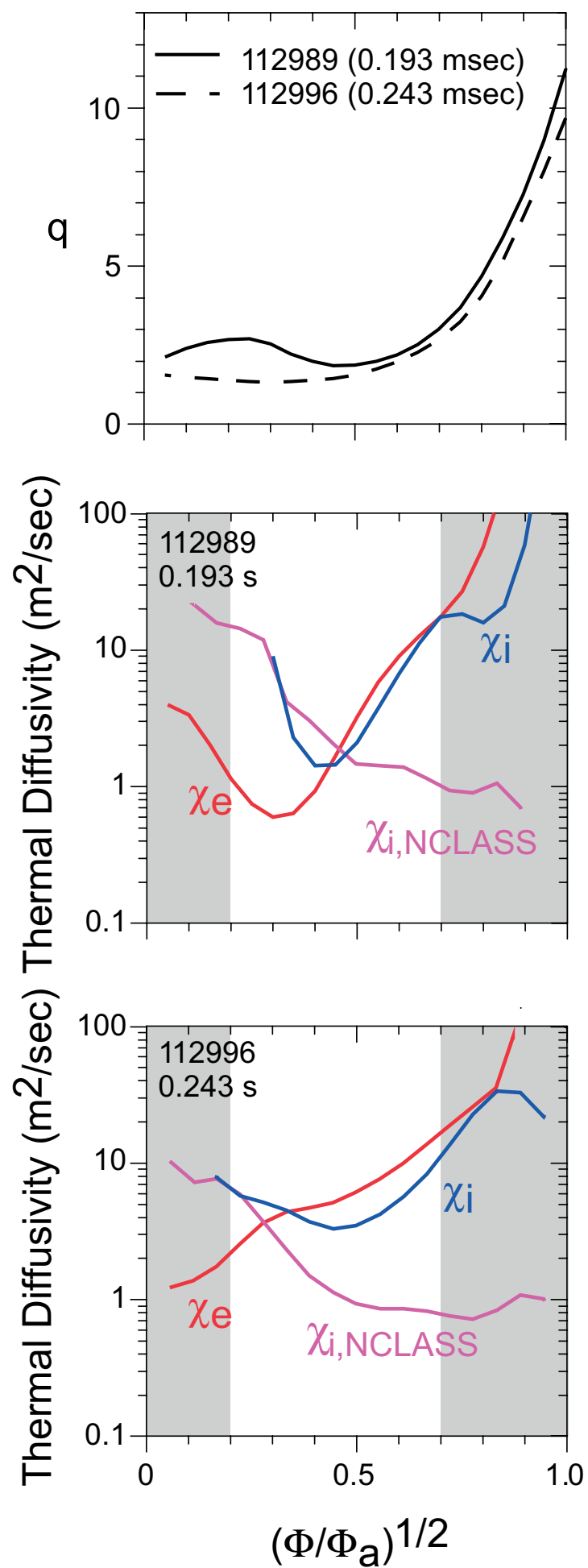

Figure 13. Radial $q$-profiles as calculated in TRANSP (top panel) and thermal diffusivities (bottom two panels) for comparison discharges, plotted as a function of the local toroidal flux, $\Phi$, normalized to the toroidal flux at the plasma boundary, $\Phi_{\mathrm{a}}$.

STs, other techniques to accomplish this must be developed. The electron Bernstein wave (EBW) is one candidate. In this approach, a launched O-mode wave is converted to an EBW, which then heats the electrons locally at the cyclotron layer in the perpendicular direction. Modelling for high- $\beta_{\mathrm{T}}$ NSTX equilibria indicates that off-axis co-current is driven

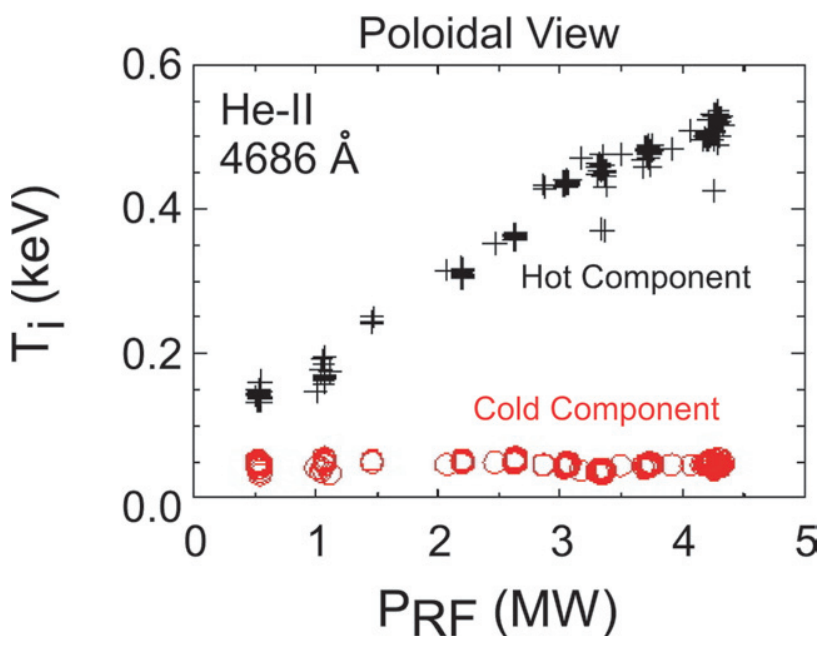

Figure 14. Edge ion temperature for the cold and hot components of the edge ions as a function of HHFW heating power.

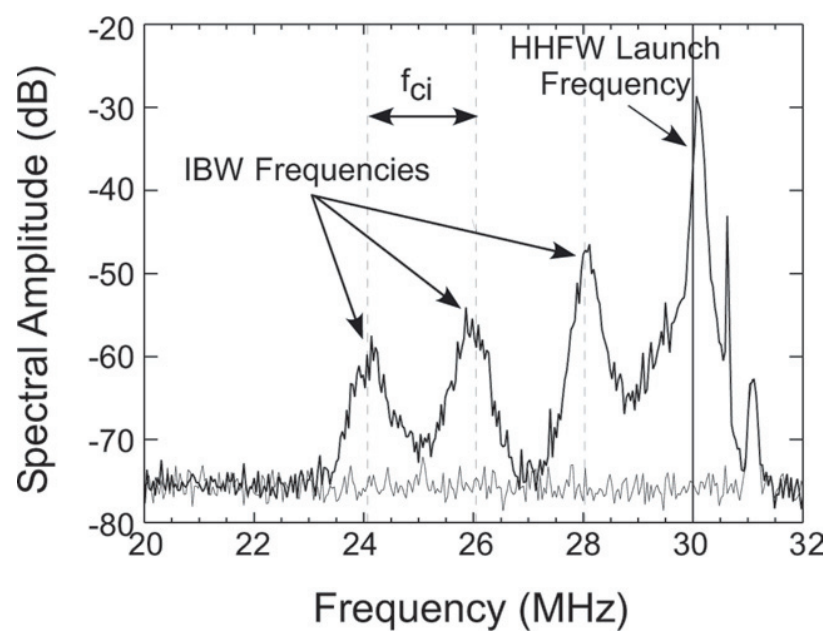

Figure 15. Parametric decay of HHFW into IBWs as measured by an RF probe. The lower curve represents the noise level of the probe.

efficiently via the Ohkawa mechanism, in which passing electrons become trapped, thus reducing counter-current drive $[32,33]$. The key to making this a viable technique is to have a $>80 \%$ conversion efficiency from O-mode to the EBW. Assessments of 16-18 GHz EBW emission and estimates of mode conversion efficiency in NSTX support this requirement, and plans for developing a $3 \mathrm{MW}, 28 \mathrm{GHz} \mathrm{EBW}$ system are underway [33].

NSTX and STs, in general, are particularly susceptible to fast ion driven instabilities due to the intrinsically low $B_{\mathrm{T}}$. The super-Alfvénic $80 \mathrm{kV}$ neutral beam ions have similar dimensionless parameters to the $3.5 \mathrm{MeV}$ alpha particles from the D-T fusion reaction in proposed tokamak reactors. In NSTX, neutral beam heated plasmas typically exhibit a broad spectrum of energetic ion excited instabilities excited through a resonant interaction with fast ions [34]. At the highest frequencies $\left(0.3<\omega / \omega_{\mathrm{ci}}<1\right)$ are the compressional and global Alfvén waves (CAE and GAE). At intermediate frequencies $(\simeq 100 \mathrm{kHz})$ are a form of shear Alfvén waves, the toroidal Alfvén eigenmodes (TAE). These are weakly damped, 
natural plasma modes whose frequency is determined largely by the thermal plasma parameters. For the large fast ion $\beta_{\mathrm{T}}$ found in NSTX, there can also be energetic particle modes, instabilities whose frequency is set by parameters of the fast ion distribution, typically at frequencies below $100 \mathrm{kHz}$. Often, the presence of these modes leads to the loss of fast ions. Enhanced fast ion losses have been correlated with both the TAE-like and fishbone-like modes, but there is no observed degradation in performance (e.g. plasma stored energy and confinement) correlated with the appearance of CAE activity. There are some indications that the CAE modes do affect the fast ion distribution. Bursts of CAE activity in some cases appear to trigger the growth of the lower frequency fast ion driven instabilities, explainable by CAE-induced transport of fast ions in either real or velocity space.

\subsection{Plasma-boundary interface}

The exploration of improved particle control and plasma fuelling benefitted from the implementation of several new techniques and capabilities. Boronization during $350^{\circ} \mathrm{C}$ bakeout, deposition of 1-2 $\mathrm{g}$ of trimethylboron prior to a run day and interspersing plasma and helium conditioning discharges all helped to maintain good wall conditions and led to better density control. Initial experiments were successfully performed using a Li pellet injector and a supersonic gas injector for localized and efficient fuelling. The use of these capabilities and techniques will be expanded in future operation [35].

Because of the compact nature of the ST, it is important to not only account for the power escaping from the plasma but to reduce the power to the material surfaces. Power accountability in both LSN and DND plasmas was found to be good [36], with up to $70 \%$ and $90 \%$ of the power being accounted for in the two configurations, respectively. The largest fraction of the power loss $(35 \%)$ was deposited on the divertor plates, with an out-in ratio of up to $5: 1$. Inner divertor detachment was found to reduce the power loading of the inner divertor plates to values of $<1 \mathrm{MW} \mathrm{m}^{-2}$ [37]. Inner divertor detachment was observed in both L- and $\mathrm{H}$-mode NBI-heated plasmas at densities $>2 \times 10^{19} \mathrm{~m}^{-3}$. Figure 16 shows an example of the inner divertor detachment in an ELM-free H-mode; a cold, dense highly recombining and highly radiating MARFE-like plasma region developed on the inner divertor plate at $0.12 \mathrm{~s}$, as indicated by a sharp increase in the peak and average $\mathrm{D}_{\gamma} / \mathrm{D}_{\alpha}$ ratios, an increase of the inner divertor radiation (bottom panel) and the appearance (not shown) of Stark-broadened Balmer series lines originating from the high atomic levels 5-10. The increasing divertor radiation with time, despite the reduction in the $\mathrm{D}_{\gamma} / \mathrm{D}_{\alpha}$ ratios, indicates that volume recombination is still occurring and that the inner divertor remains detached. The outer divertor in all experiments remained attached, with heat fluxes up to $10 \mathrm{MW} \mathrm{m}^{-2}$ [38].

A variety of ELMs, which can cause transient increased divertor power loading, was observed in H-mode plasmas. An apparently new type of ELM, Type V, was identified in LSN discharges $[39,40]$. This ELM is small amplitude with minimal energy loss and minimal resulting power loading. These ELMs occur in plasmas in the collisionality regime $\nu_{\mathrm{e}}^{*}>1$, where $\nu_{\mathrm{e}}^{*}$ is evaluated at the top of the density pedestal. At lower $\nu_{\mathrm{e}}^{*}$, this small ELM was interspersed between large Type I

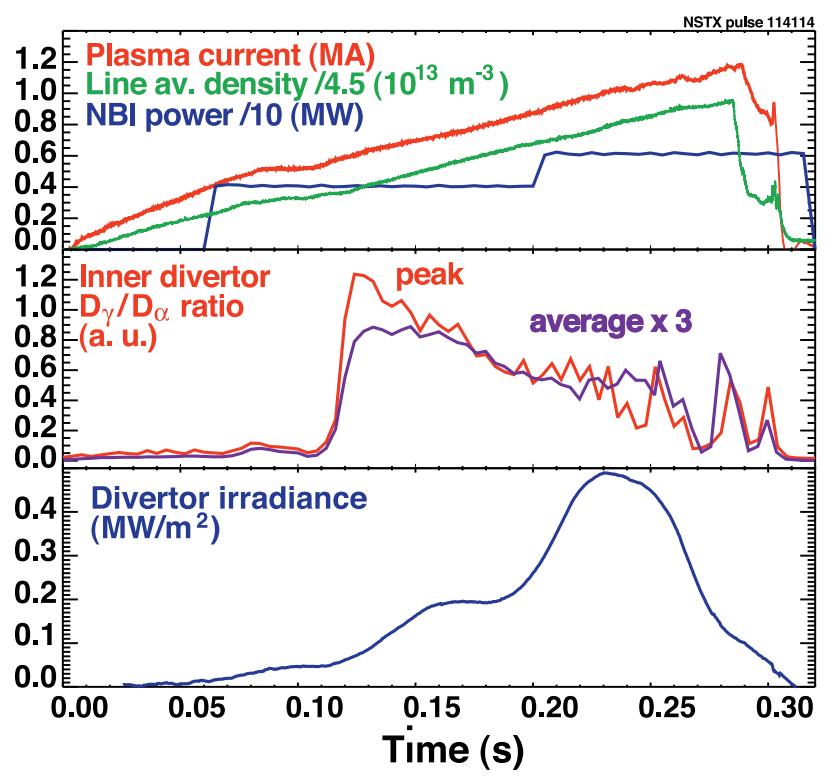

Figure 16. Time evolution of a plasma discharge showing inner divertor detachment. The middle panel shows peak and average $\mathrm{D}_{\gamma} / \mathrm{D}_{\alpha}$ ratios, and the bottom panel is a measure of divertor radiation.

ELMs. The Type V ELM originates in the lower part of the vessel, near the lower X-point, which is in the direction of the ion $\nabla B$ drift, and it propagates poloidally in the electron diamagnetic direction. Type I ELMs often exhibited low- $n$ external kink-like structures on the fast camera images, while structures associated with Type II/III/V ELMs were higher $n$. The severity of ELMs that affect the plasma stored energy was found to depend sensitively on plasma elongation. Future work will focus on understanding the underlying ELM stability properties and their dependence on shape, using high spatial resolution edge diagnostics, in order to utilize them for control of both density and power loading.

The two-dimensional structure of edge plasma turbulence was measured by viewing the emission of $\mathrm{D}_{\alpha}$ or helium spectral lines enhanced by gas puffing using an ultra-high speed CCD camera [41]. Transitions from L-mode to H-mode could appear as a continuous evolution from a turbulent 'blob-like' or intermittent state to a quiescent state over $0.1 \mathrm{~ms}$, apparently without any new spatial features or flows. Transitions from $\mathrm{H}$-mode to L-mode appeared as high- $n$ poloidal perturbations, which evolved into radially moving blobs. ELMs normally were associated with an increase in blob-like activity, although sometimes ELM-free H-modes had intermittent blob-like turbulence. Blobs are regions of enhanced light emission that form near the separatrix and move outwards. The underlying physics of their origin and formation, however, is not known.

\subsection{Integrated high performance}

The performance enhancements achieved in each of the individual physics areas have been integrated to produce high- $\beta_{\mathrm{T}}$ plasmas with a significant amount of non-inductive driven current. Figure 17 reflects this progress statistically, where an estimate of bootstrap current fraction $\left(\epsilon^{1 / 2} \beta_{\mathrm{P}} / 2\right.$, where $\epsilon$ is the inverse aspect ratio, $a / R)$ is plotted against $\beta_{\mathrm{T}}$ for NSTX discharges from the past four years of operation. 
Progress towards high performance plasmas in NSTX

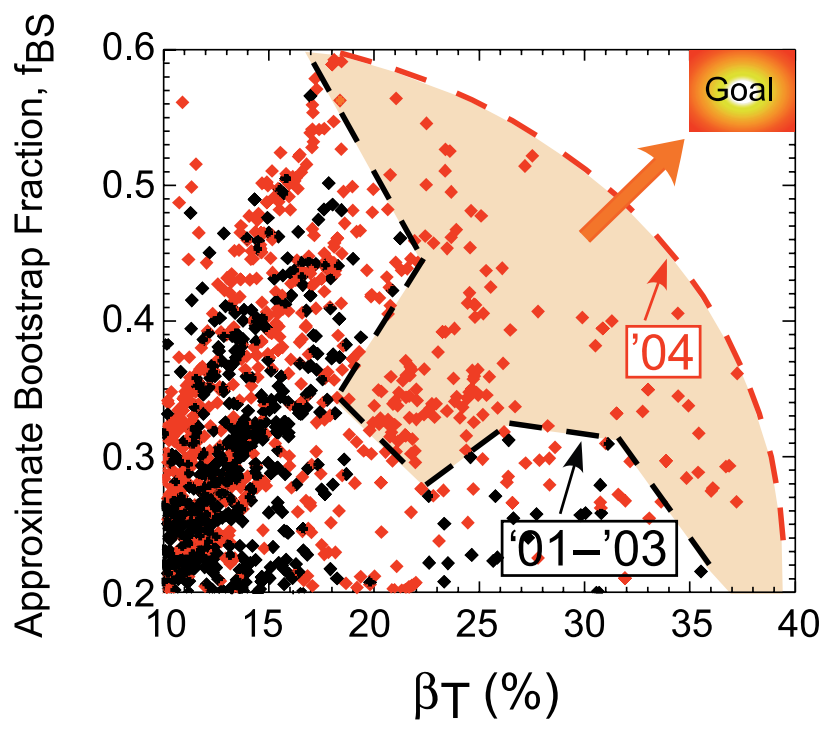

Figure 17. A crude estimate of bootstrap fraction plotted as a function of $\beta_{\mathrm{T}}$ for discharges from 2004 (red points) and those from earlier years (black points).

The target of high non-inductive fraction $(\sim 60 \%)$ and high $\beta_{\mathrm{T}}(\sim 40 \%)$ is shown in the upper right-hand corner. The red points were data taken during the 2004 experimental campaign, and the black points were taken prior to this year. As can be seen, a large step in both directions (shaded region) has been taken towards the target, with a significant number of discharges with both $\beta_{\mathrm{T}}>20 \%$ and with $>40 \%$ estimated bootstrap fraction. The progress made during the 2004 campaign is indicated by the shaded region.

A specific example of such an integrated highperformance discharge at $B_{\mathrm{T}}=0.44 \mathrm{~T}$ is shown in figure 18 . The improved shaping capability $(\kappa=2.3)$ and early $\mathrm{L}-\mathrm{H}$ transition in this discharge led to lower $l_{\mathrm{i}}$ and reduced Ohmic flux consumption. This $1 \mathrm{MA}$ discharge was heated by over $7 \mathrm{MW}$ of NBI. During the H-phase, the $Z_{\text {eff }}$ in the core of the plasma increased in time from approximately 1.5 to 3 , while that near the edge remained high, near 5 , due to the buildup of carbon there. The discharge had a current flat-top time of $0.8 \mathrm{~s}$, which is approximately four current relaxation times. According to model calculations, the $q$-profile increased monotonically with the radius, but remained approximately constant for the last $300 \mathrm{~ms}$ of the discharge ( $>1$ current relaxation time) with $q_{0}$ approximately 1 . The stored energy of the plasma plateaued at $280-300 \mathrm{~kJ}$ and $\beta_{\mathrm{T}}$ at $>20 \%$ for approximately $0.5 \mathrm{~s}$, which is over ten energy confinement times. $\beta_{\mathrm{N}}$ exceeded 5 and $\tau_{E} / \tau_{97 \mathrm{~L}}$ was about 1.7 simultaneously for the same duration. The line-averaged density exhibited only a modest increase after $t=0.3 \mathrm{~s}$, and was then held constant at $80 \%$ of the Greenwald limit by low ELM activity. $n_{\mathrm{e}} / n_{\mathrm{GW}}$ reached $0.9-1$ in other discharges, and minimal confinement degradation was observed at these high normalized densities.

In this and similar discharges, the loop voltage remained low $(<0.5 \mathrm{~V})$ through the duration of the current and energy flat-top, indicating a significant amount of non-inductive driven current. The fractions of current driven by various sources are plotted as a function of time in figure 19 for a discharge similar to, but of shorter duration than, the one shown

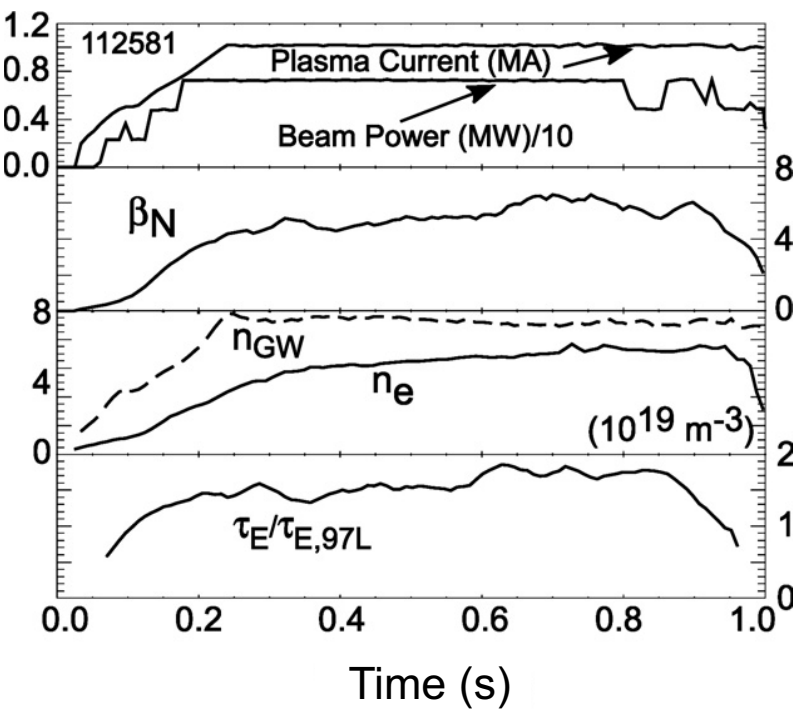

Figure 18. Time evolution of a long-pulse, high-performance discharge. This discharge had $\kappa=2.3$ and a hollow $Z_{\text {eff }}$ profile with values of near 5 at the edge and a value of 1.5 , increasing to 3 with time, in the core. The magnetic shear for this discharge, as calculated in TRANSP, was monotonically increasing with radius, but was low in the core.

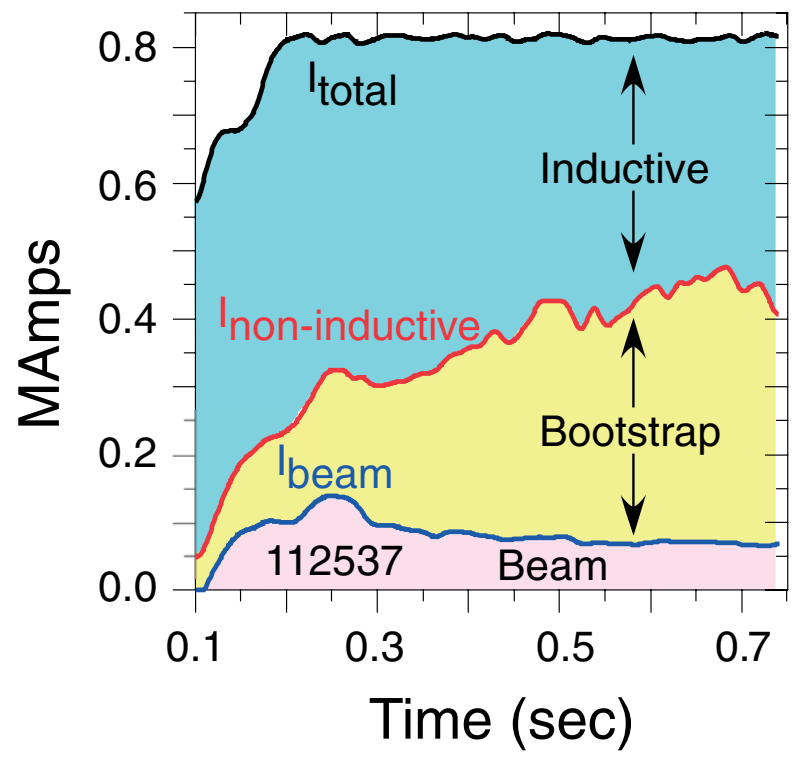

Figure 19. Time evolution of current fractions for a long-pulse, high-performance discharge similar to the one shown in the previous figure.

in figure 18 . Approximately $60 \%$ of the total current was driven non-inductively by beams (10\%) and bootstrap (50\%), as calculated by TRANSP. The MSE diagnostic, commissioned at the end of the 2004 run, will measure the current directly in order to aid in the assessment of the non-inductive current fraction and profile.

\section{Summary and future plans}

NSTX has made significant progress towards its goal of establishing the Spherical Torus physics database at high $\beta_{\mathrm{T}}$, with enhanced confinement and low ion transport levels, and 
mitigation of divertor heat loads. More routine access to high $\beta_{\mathrm{T}}$ was achieved during the last experimental campaign, along with a better understanding of the processes, from both internal and external MHD modes, that are limiting. The thermal and global confinement times are found to scale with current and power as in conventional aspect ratio tokamaks, with enhanced values relative to conventional aspect ratio scalings, but with a dependence on toroidal field. Research has focused on developing an understanding of how rotational and magnetic shear, as well as electromagnetic turbulence, affect performance. These measurements, coupled with both linear and non-linear gyrokinetic calculations, will lead to an integrated understanding of the fundamental transport processes in high- $\beta_{\mathrm{T}}$ plasmas. Despite outstanding issues in understanding HHFW power deposition and fast ion confinement, other means to both generate and sustain current non-inductively have been explored. Non-solenoidal start-up has been demonstrated by two different techniques. The advances over the past year in operational techniques to create favourable plasma profiles have led to significant progress towards the NSTX target of a high performance, non-inductively driven plasma, with frequent attainment of non-inductive current fractions of over $40 \%$ and $\beta_{\mathrm{T}}>20 \%$. Furthermore, NSTX has achieved the performance levels in $\beta_{\mathrm{T}}$ and confinement enhancement factor required for a successful Component Test Facility [2].

The next experimental campaign will take advantage of enhanced capabilities and techniques in order to progress further towards the NSTX goals. Current profile measurements will aid analysis efforts leading to a better determination of stability as well as the effect of the magnetic shear on plasma transport properties. Higher resolution edge diagnostics will also benefit transport and stability studies. A microwave scattering diagnostic designed to measure high- $k\left(k_{\theta} \rho_{\mathrm{s}} \gg 1\right)$ fluctuations will be commissioned by the end of the 2005 experimental campaign, and will help explore the role of ETG modes in electron transport. The role of rotation and possible suppression of external MHD modes will be studied using the new set of active control coils. NSTX is preparing additional technical capability for non-solenoidal start-up studies, an enhanced suite of diagnostics for HHFW coupling studies and new methodologies and technical capabilities for particle and power control.

\section{Acknowledgments}

This work was supported by the US Department of Energy Contract DE-AC02-76CH03073 at the Princeton Plasma Physics Laboratory, DE-AC05-00OR22725 at Oak Ridge National Laboratory, DE-AC04-94AL85000 at Sandia National Laboratory, W-7405-ENG-36 at Los Alamos National Laboratory, W-7405-ENG-48 at Lawrence Livermore National Laboratory, Grant DE-FG02-99ER54523 at Johns Hopkins University, DE-FG03-99ER54519 at the University of Washington, DE-FG03-99ER51069 at UC San Diego, DE-FG02-99ER54525，DE-FG02-99ER54521， DE-FG0291ER54109 at MIT, DE-FG02-99ER54524 at Columbia University, DE-FG03-02ER54684 at Compx, DE-FG0299ER54520 at Nova Photonics, DE-FG02-99ER54518 at
UC Davis, DE-FG03-99ER54527 at UCLA, DE-FG0299ER54522 at General Atomics, DE-FG03-02ER54681 at UC Irvine, DE-FG02-93ER54215 at University of Rochester, DEFG02-86ER53223 at NYU and DE-FG02-01ER54619 at University of Wisconsin.

\section{References}

[1] Ono M. et al 2004 Nucl. Fusion 44452

[2] Peng M. et al 2005 Physics and engineering assessments of spherical torus component test facility Nucl. Fusion submitted

[3] Ono M. et al 2003 Plasma Phys. Control. Fusion 45 A335

[4] Synakowski E.J. et al 2003 Nucl. Fusion 431653

[5] Bell M.E. et al 2004 Physics results from the National Spherical Torus Experiment Proc. 31st EPS Conf. on Plasma Physics (London, 2004) vol 28G (Europhysics Conference Abstracts) ed P. Norreys and H. Hutchinson (Geneva: European Physical Society) paper P2.194 (on CD-ROM)

[6] Lloyd B. et al 2003 Nucl. Fusion 431665

[7] Raman R. et al 2004 Phys. Plasmas 112565

[8] Gartsak G. et al 2003 Phys. Plasmas 101705

[9] Mueller D. et al 2004 Real-time equilibrium reconstruction and isoflux control of plasma shape and position in the National Spherical Torus Experiment NSTX Proc. 31st EPS Conf. on Plasma Physics (London, 2004) vol 28G (Europhysics Conference Abstracts) ed P. Norreys and H. Hutchinson (Geneva: European Physical Society) paper P2.199 (on CD-ROM)

[10] Ferron J.R. et al 1998 Nucl. Fusion 381055

[11] Boedo J. et al 1998 Rev. Sci. Instrum. 692663

[12] Lao L.L. et al 1985 Nucl. Fusion 251611

[13] Sabbagh S.A. et al 2001 Nucl. Fusion 411601

[14] Gates D.A. et al 2003 Phys. Plasmas 101659

[15] Wade M.R. et al 2003 Nucl. Fusion 43634

[16] Menard J. et al 2005 Nucl. Fusion 45 539-56

[17] Sabbagh S.A. et al 2005 Wall stabilized operation in high beta NSTX plasmas Nucl. Fusion submitted

[18] Glasser A.H. and Chance M.S. 1997 Bull. Am. Phys. Soc. 42 1848

[19] Meyer H. et al $2005 \mathrm{H}$-mode transition physics close to double null on MAST and its application to other tokamaks $\mathrm{Nucl}$. Fusion submitted

[20] Whyte D.G. et al 1997 Nucl. Fusion 38387

[21] Kaye S.M. et al 1997 Nucl. Fusion 371303

[22] ITER Physics Basis 1999 Nucl. Fusion 392137

[23] Rhodes T.L. et al 2002 Phys. Plasmas 92141

[24] LeBlanc B.P. et al 2004 Nucl. Fusion 44513

[25] Houlberg W. et al 1997 Phys. Plasmas 43230

[26] Gates D.A., White R. and Mynick H. 2004 Phys. Plasmas $11 \mathrm{~L} 45$

[27] Stutman D. et al 2005 Studies of improved electron confinement in NSTX Nucl. Fusion submitted

[28] Sauter O. et al 1999 Phys. Plasmas 62834

[29] Kotschenreuther M. et al 1995 Comput. Phys. Commun. 88128

[30] Dorland W. et al 2000 Phys. Rev. Lett. 855579

[31] Biewer T. et al 2004 Rev. Sci. Instrum. 75650

[32] Ohkawa T. 1976 General Atomics Report GA-A13847

[33] Taylor G. et al 2004 Phys. Plasmas 114733

[34] Fredrickson E. et al 2005 Study of aspect ratio effects on kinetic MHD instabilities in NSTX and DIII-D Nucl. Fusion submitted

[35] Kugel H. et al 2005 J. Nucl. Mater. 337-339 495

[36] Paul S.P. et al 2005 J. Nucl. Mater. 337-339 251

[37] Soukhanovskii V. et al 2005 J. Nucl. Mater. 337-339 475.

[38] Maingi R. et al 2003 Nucl. Fusion 43969

[39] Maingi R. et al 2005 Nucl. Fusion 45 1066-77

[40] Maingi R. et al 2005 J. Nucl. Mater. 337-339 727

[41] Zweben S.J. et al 2004 Nucl. Fusion 44134 